\title{
Monodromy- and Spectrum-Preserving Deformations I
}

\author{
Hermann Flaschka ${ }^{\star}$ and Alan C. Newell \\ Department of Mathematics and Computer Science, Clarkson College of Technology, Potsdam \\ N. Y. 13676 USA
}

\begin{abstract}
A method for solving certain nonlinear ordinary and partial differential equations is developed. The central idea is to study monodromy preserving deformations of linear ordinary differential equations with regular and irregular singular points. The connections with isospectral deformations and with classical and recent work on monodromy preserving deformations are discussed. Specific new results include the reduction of the general initial value problem for the Painleve equations of the second type and a special case of the third type to a system of linear singular integral equations. Several classes of solutions are discussed, and in particular the general expression for rational solutions for the second Painleve equation family is shown to be $-\frac{d}{d x} \ln \left(\Delta_{+} / \Delta_{-}\right)$, where $\Delta_{+}$and $\Delta_{-}$are determinants. We also demonstrate that each of these equations is an exactly integrable Hamiltonian system. The basic ideas presented here are applicable to a broad class of ordinary and partial differential equations; additional results will be presented in a sequence of future papers.
\end{abstract}

\section{Introduction and Outline}

This paper is the first in what is planned to be a series of studies on deformations of linear ordinary differential equations with coefficients rational on a Riemann surface. The deformations in question preserve the monodromy at singular points of the linear equation, and this requirement forces the coefficients of the linear equation to satisfy certain nonlinear ordinary or partial differential equations of considerable interest. The theory of monodromy-preserving deformations overlaps the theory of isospectral deformations (i.e., soliton theory), and indeed one of our aims will be to understand the connections between these two types of problems. Applications of the nonlinear equations governing monodromypreserving deformations have been discovered in nonlinear waves, statistical

* Permanent address: Mathematics Department, University of Arizona, Tucson, AZ 85721 USA 
mechanics and quantum field theory; we shall present some new results along these lines, and we intend to develop relations between soliton ideas and the applications mentioned above.

The next section of this paper will describe in considerable detail the history of relevant work on monodromy-preserving deformations, as well as connections between different approaches and problems. The paper itself deals primarily with two representative examples. The first of these is the system

$$
\begin{aligned}
& v_{1 \zeta}=-i\left(4 \zeta^{2}+x+2 q^{2}\right) v_{1}+(4 \zeta q-2 i r+v / \zeta) v_{2} \\
& v_{2 \zeta}=(4 \zeta q+2 i r+v / \zeta) v_{1}+i\left(4 \zeta^{2}+x+2 q^{2}\right) v_{2},
\end{aligned}
$$

with $x, q, r, v$ constant in $\zeta$. The singular points of (1.1) are at $\zeta=\infty$ (irregular) and $\zeta=0$ (regular) if $v \neq 0$. A deformation of (1.1) is monodromy-preserving if the Stokes multipliers associated with formal solutions about $\zeta=\infty$, the monodromy matrix about $\zeta=0$, and the matrix connecting fundamental solutions at $\zeta=0$ and $\infty$ are unchanged (when $x$ is varied, and $q, r$ change as functions of $x$ ). For this it is necessary and sufficient that (as function of $x$ ) $r=q_{x}$, and

$$
q_{x x}=2 q^{3}+x q-v .
$$

(1.2) is the second Painleve equation [1]. By posing an irregular-singular RiemannHilbert problem, we exploit the connection with (1.1) to reduce (1.2) to an equivalent system of linear singular integral equations. In a special case, this reproduces the solution, due to Ablowitz and Segur, of (1.2) by a Marchenko integral equation [2]. Other special cases yield the rational or Airy-function solutions of (1.2) discovered by Airault [3], but this time by procedures very familiar from the inverse-scattering derivation of multisoliton formulae.

The second and somewhat more complicated example, whose study we begin in this paper, is afforded by the system

$$
\begin{aligned}
& v_{1 \zeta}=\left(-i x+\frac{i}{\zeta^{2}} \cosh u\right) v_{1}+\left(-\frac{x u_{x}}{2 \zeta}+\frac{i}{\zeta^{2}} \sinh u\right) v_{2}, \\
& v_{2 \zeta}=\left(-\frac{x u_{x}}{2 \zeta}-\frac{i}{\zeta^{2}} \sinh u\right) v_{1}+\left(i x-\frac{i}{\zeta^{2}} \cosh u\right) v_{2} .
\end{aligned}
$$

This system has irregular singular points at $\zeta=0, \infty$. The deformation equation is equivalent to a special case of the third Painlevé equation,

$$
\left(x u_{x}\right)_{x}=-4 \sinh u .
$$

This equation and its linearization via the Riemann-Hilbert problem for (1.3) provide a link between the inverse scattering transform and the extensive work of Barouch-McCoy-Tracy-Wu [4-6] on the Ising model and of Sato et al. [7] on monodromy-preserving deformations and quantum field theory.

Our principal aims in this first paper are:

1. To point out relations between mondromy - and spectrum - preserving deformations.

2. To introduce a new method for solving the initial value problem for equations such as (1.2) and (1.4) which can be written as monodromy preserving deformations. 
3. To point out that both (1.2) and (1.4) are exactly integrable Hamiltonian systems.

4. To discuss the connections between our method, the inverse scattering transform and the novel ideas of Krichever and Novikov for investigating multiperiodic solutions of soliton equations.

Following a general discussion in Sect. 2, in Sects. 3 and 4 we describe in detail the mapping from the coefficients of the differential equations (1.1) and (1.3) to the monodromy data and prove that this data is independent of $x$. In each case we also derive the inverse mapping which allows one to reconstruct the solutions to (1.1) and (1.3) (and therefore both the equations they satisfy and the coefficients in these equations, the quantities of interest) from a knowledge of the monodromy data. The result appears as a set of coupled linear singular integral equations. We examine several limiting cases and derive some useful formulae for special classes of solutions, although we have not, as yet, been able to prove the existence and uniqueness of solutions in the general case.

\section{Introduction and Discussion}

\section{A. Deformations}

Nonlinear ordinary and partial differential equations do not, in general, admit explicit solutions, because the solutions of the typical nonlinear equation are so wildly irregular that they could not possibly be represented by known functions. Conversely, nonlinear equations with very well-behaved solutions should be expected to have uncommon properties. One feature shared by many such special nonlinear equations was discovered towards the end of the 19 th century, was exploited for about forty years and then (apparently) forgotten; quite recently it has re-emerged in a somewhat different form. This is the observation that "solvable" nonlinear equations arise as integrability conditions for certain kinds of deformations of linear equations.

The most recent version of this technique centers on the idea of isospectral deformation. The best known and most celebrated example involves the Schrödinger operator [8] $L=-\frac{d^{2}}{d x^{2}}+q(x)$. One asks, how can one deform the coefficient $q(x)$ as a function of an additional parameter $t$, so that the eigenvalues of $L$ [as operator on $L^{2}(R)$, say] do not change? The simplest nontrivial such deformation is already one of great physical importance: the deformation is isospectral if $q(x, t)$ satisfies the Korteweg-de Vries equation,

$$
q_{t}-6 q q_{x}+q_{x x x}=0
$$

In recent years, studies of isospectral deformations have uncovered many more nonlinear equations of physical relevance and mathematical interest; the whole subject appears to be deeply involved with Lie groups and differential and algebraic geometry.

Another kind of deformation, perhaps not as widely known, is associated with the monodromy group of Fuchsian differential equations. Consider a (matrix) 
system of ordinary differential equations,

$$
Y^{\prime}=Y \sum_{j=1}^{n} \frac{A^{j}}{\zeta-a_{j}},
$$

where the $A^{j}$ are constant $m \times m$ matrices. The fundamental solution of (2.2) is (in general) a multi-valued function of complex $\zeta$. If $\zeta$ moves on a path $\left|\zeta-a_{j}\right|=\varepsilon$ encircling $a_{j}$, the solution $Y\left(a_{j}+\varepsilon\right)$ will change into $Y\left(a_{j}+\varepsilon e^{2 \pi i}\right)=\tilde{Y}$ which is a matrix whose rows are linear combinations of the rows of $Y(\zeta)$,

$$
\tilde{Y}(\zeta)=M_{j} Y(\zeta)
$$

$M_{j}$ is the monodromy matrix at $a_{j}$. The deformation problem is: how can one change the $A^{j}$, as function of the poles $a_{j}$, so that the monodromy matrices $M_{j}$ remain fixed? The simplest nontrivial example is again of considerable interest. The linear Eq. (2.2) is taken to be a $2 \times 2$ system, with three poles fixed at $0,1, \infty$, and one pole $\tau$ subject to variation. $A$ priori, there are twelve adjustable entries in the coefficient matrices $A^{j}(j=1,2,3)$, but they can all be expressed in terms of a single function $z(\tau)$ which satisfies the equation $[9,18]$

$$
\begin{aligned}
z^{\prime \prime}+ & {\left[\frac{1}{\tau}+\frac{1}{\tau-1}+\frac{1}{z-\tau}\right] z^{\prime}-\frac{1}{2}\left[\frac{1}{z}+\frac{1}{z-1}+\frac{1}{z-\tau}\right]\left(z^{\prime}\right)^{2}-2 \frac{z(z-1)(z-\tau)}{\tau^{2}(\tau-1)^{2}} } \\
\cdot & \left\{\alpha-\beta \frac{\tau}{z^{2}}+\gamma \frac{\tau-1}{(z-1)^{2}}-\delta \frac{\tau(\tau-1)}{(z-\tau)^{2}}\right\}=0 .
\end{aligned}
$$

The frightening Eq. (2.4) is the most general 2 nd order equation

$$
z^{\prime \prime}=R\left(\tau, z, z^{\prime}\right)
$$

with $R$ rational in $z, z^{\prime}$ and analytic in $\tau$, which has the property: the location of any algebraic, logarithmic, or essential singularity of its solutions is independent of the initial conditions.

Equations with this property were studied in exhaustive (and exhausting) detail by Painlevé and Gambier [1]. There are fifty canonical types, which include linear equations such as $z^{\prime \prime}=z$, equations solved by elliptic functions, such as

$$
z^{\prime \prime}=2 z^{3}+c z-v
$$

and six equation types whose general solutions can be proved not to be expressible in terms of the basic special functions (except for isolated cases, see Sect. 3 below). These six equations are called Painlevé equations, and their solutions Painlevé transcendents. These equations are, as the summary above indicates, distinguished among non-classical ordinary differential equations of the form (2.4) in that the nonpolar singularities of their solutions can be predicted from the equation alone. Equations (1.2) and (1.4), which we study in this paper, fall into this class $[(1.4)$ only after the change of variables $\left.f=e^{u}\right]$. We shall describe later some of the important applications of the Painleve transcendents.

A third kind of deformation involves the properties of solutions of ordinary differential equations near irregular singular points. This, indeed, will be the main concern of the present paper, and for the moment we provide a brief description 
only. Consider the system

$$
Y^{\prime}=A(\zeta) Y \text {, }
$$

where the (matrix) function $A$ is polynomial in $\zeta . \zeta=\infty$ is an irregular singular point, and typically only formal solutions

$$
\tilde{Y}(\zeta)=e^{P(\zeta)} \zeta^{l}\left\{1+c_{1} \zeta^{-1}+c_{2} \zeta^{-2}+\ldots\right\},
$$

are available near $\infty$. A formal series such as (2.7) is, in fact, asymptotic to a true solution $Y$ in certain sectors $S$ in the complex $\zeta$-plane, and one may have

$$
\begin{aligned}
& Y_{j} \sim \tilde{Y} \text { in } S_{j} \\
& Y_{k} \sim \tilde{Y} \text { in } S_{k}
\end{aligned}
$$

for different true solutions $Y_{j}, Y_{k}$ in different sectors $S_{j}, S_{k}$. The discontinuity of asymptotic expansions is known as the Stokes phenomenon, and the matrices which connect the different true solutions with fixed asymptotic expansions in the various sectors are called Stokes multipliers, $Y_{j}=Y_{k} M_{k j}$. The deformation problem we pose is: to change the coefficients in (2.6) so that the Stokes multipliers $M_{k j}$ remain constant.

The immediate object of our paper is to study this deformation problem for a particular $2 \times 2$ system which leads to the second Painlevé equation

$$
q^{\prime \prime}=2 q^{3}+x q-v
$$

as integrability condition. (2.8) is a nonautonomous version of the elliptic function Eq. (2.5). We will reduce this nonlinear ordinary differential equation to a system of linear integral equations, and in the process we will recover some known special solutions. There are, however, deep and entirely unexplored connections amongst these various types of deformation problems, and equally interesting relations between monodromy-preserving deformations and questions in statistical mechanics, quantum field theory, and wave dynamics. We plan to address some of these topics in later papers, and want to detail in this overall introduction the ingredients of what we think will eventually become a beautiful and useful complement to current soliton theory.

\section{B. Applications}

Painlevé transcendents are encountered in several important physical problems, of which we describe two. In one of these, the connection with solitons (more precisely, with isospectral deformations) is evident. In the other, there are certain analogies with soliton theory which first stimulated our interest. We now outline the relevant facts.

I. Self-Similar Solutions of Wave Equations. Although the following considerations apply to many soliton equations, we restrict ourselves to the modified Kortewegde Vries (MKdV) equation, in the form

$$
q_{t}-6 q^{2} q_{x}+q_{x x x}=0 \text {. }
$$


If $q(x, t)$ solves $(2.9)$, then so does

$$
\tilde{q}(x, t)=\beta q\left(\beta x, \beta^{3} t\right)
$$

for any $\beta$. A solution which is invariant under this scaling is called self-similar: $q(x, t)=\tilde{q}(x, t)$. It follows that for such $q$,

$$
q(x, t)=(3 t)^{-1 / 3} f\left(x(3 t)^{-1 / 3}\right) .
$$

The function $f(\xi)$ satisfies

$$
f^{\prime \prime}=2 f^{3}+\xi f-v
$$

( $v$ is an arbitrary integration constant), as can be seen by substitution of (2.10) into (2.9). (2.11) is the second Painlevé equation. Now, it is known that MKdV can be integrated by the inverse-scattering problem for

$$
\begin{aligned}
& v_{1 x}+i \zeta v_{1}=q v_{2} \\
& v_{2 x}-i \zeta v_{2}=q v_{1}
\end{aligned}
$$

with the $t$-evolution of the eigenfunctions governed by

$$
\begin{aligned}
& v_{1 t}=\left(-4 i \zeta^{3}-2 i q^{2} \zeta\right) v_{1}+\left(4 \zeta^{2} q+2 i \zeta q_{x}-q_{x x}+2 q^{3}\right) v_{2}, \\
& v_{2 t}=\left(4 \zeta^{2} q-2 i \zeta q_{x}-q_{x x}+2 q^{3}\right) v_{1}+\left(4 i \zeta^{3}+2 i q^{2} \zeta\right) v_{2} .
\end{aligned}
$$

For the self-similar solution (2.10), the $t$ equation is in a sense redundant, since $q(x, t)$ is known for all $t$ once it is given for $t=1 / 3$, say $(q(x, 1 / 3)=f(x))$. This observation can be put to use in two ways.

Ablowitz and Segur [10] in their analysis of the asymptotic behavior of solutions of the $\mathrm{KdV}$ equation were led to the following procedure (described here for $\mathrm{MKdV}$ ). Apply the usual inverse method for MKdV up to the Marchenko equation by which $q(x, t)$ is determined from the scattering data. At that stage, assume $q(x, t)$ to be self-similar, and observe that $t$ can be scaled out of the Marchenko equation altogether. This leads to the Fredholm equation,

$$
\begin{aligned}
& K_{1}(x, y)=\varrho \mathrm{Ai}\left(\frac{x+y}{2}\right)-\frac{1}{2} \varrho \int_{x}^{\infty} K_{2}(x, s) \mathrm{Ai}\left(\frac{s+y}{2}\right) d s \\
& K_{2}(x, y)=-\frac{1}{2} \varrho \int_{x}^{\infty} K_{1}(x, s) \mathrm{Ai}\left(\frac{s+y}{2}\right) d s
\end{aligned}
$$

and one recovers the Painlevé transcendent $f(x)$ by

$$
f(x)=K_{1}(x, x) .
$$

It is a consequence of the scaling invariance of the Marchenko equation that the reflection coefficient $r(\zeta)$ has the special form

$$
r(\zeta)=\varrho e^{8 i \zeta^{3 / 3}} .
$$

The Fourier transform of $R(\zeta)$ is the kernel of (2.14), which explains the occurrence of the Airy function. This method produces the unique solution of (2.11) which satisfies

$$
f(x) \sim \varrho \operatorname{Ai}(x), \quad x \rightarrow+\infty
$$


(Hastings and McLeod [11]). More recently, Ablowitz et al. [12] have shown how to bypass the $t$-evolution in linearizing (2.11) and similar equations for solutions which decay at $+\infty$. This is more satisfactory, since scattering theory is not really applicable for potentials such as $f(x)$ which have poles, or at least decay slowly at + or $-\infty$.

It is also possible to use the scaling invariance directly on the $t$-equation (2.13). If $\mathbf{v}(x, t, \zeta)$ solves $(2.12),(2.13)$, then so does $\mathbf{v}\left(\beta x, \beta^{3} t, \beta^{-1} \zeta\right)$ for the potential $\tilde{q}$. Define, in the case of self-similar $q$,

$$
\mathbf{w}(x, \zeta)=\mathbf{v}\left(x, \frac{1}{3}, \zeta\right)
$$

then

$$
\mathbf{v}(x, t, \zeta)=\mathbf{w}\left(x(3 t)^{-1 / 3}, \zeta(3 t)^{1 / 3}\right) .
$$

Hence

$$
\frac{\partial \mathbf{v}}{\partial t}=-(3 t)^{-4 / 3} \frac{\partial \mathbf{w}}{\partial x}+(3 t)^{-2 / 3} \zeta \frac{\partial \mathbf{w}}{\partial \zeta},
$$

and upon using the $x$-equation $(2.12)$ to eliminate $\frac{\partial \mathbf{w}}{\partial x}$, one can rewrite (2.13) as follows [we replace $x(3 t)^{-1 / 3}$ by $x$, and $\zeta(3 t)^{1 / 3}$ by $\zeta$ ]:

$$
\begin{aligned}
& w_{1 \zeta}=-i\left(4 \zeta^{2}+x+2 f^{2}\right) w_{1}+\left(4 \zeta f+2 i f^{\prime}\right) w_{2} \\
& w_{2 \zeta}=\left(4 \zeta f-2 i f^{\prime}\right) w_{1}+i\left(4 \zeta^{2}+x+2 f^{2}\right) w_{2} .
\end{aligned}
$$

This is coupled to (2.12), rewritten now without use of $t$ :

$$
\begin{aligned}
& w_{1 x}+i \zeta w_{1}=f w_{2} \\
& w_{2 x}-i \zeta w_{2}=f w_{1} .
\end{aligned}
$$

If one imagines $f(x)$ to decay so rapidly at $\pm \infty$ that scattering theory can be applied, one looks at a solution of (2.18) which satisfies

$$
\mathbf{w}(x, \zeta) \sim\left(\begin{array}{l}
1 \\
0
\end{array}\right) e^{-i \zeta x}, \quad x \rightarrow-\infty .
$$

At $+\infty$

$$
\mathbf{w}(x, \zeta) \sim a(\zeta)\left(\begin{array}{l}
1 \\
0
\end{array}\right) e^{-i \zeta x}+b(\zeta)\left(\begin{array}{l}
0 \\
1
\end{array}\right) e^{i \zeta x}
$$

and it follows from (2.17) that

$$
r(\zeta)=\frac{b(\zeta)}{a(\zeta)}=\varrho e^{8 i \zeta^{3} / 3}
$$

Inverse scattering then reproduces the results of Ablowitz and Segur. There is a conceptual question, which leads to the main point of our paper. In MKdV theory, the $x$-equation (2.12) is basic. It is deformed in $t$ in a special way; namely, so that the transmission coefficient $a(\zeta)^{-1}$ is independent of $t$. The $t$-evolution (2.13) is one possible expression of this requirement, and $\mathrm{MKdV}$ is the integrability condition 
for these two systems. For self-similar solutions, however, there is no $t$ in (2.18), so what is being deformed? As we explain in this paper, it is now the $\zeta$-equation (2.17) (alias $t$-evolution) which is basic. Vary $x$ in (2.17), and change the remaining coefficients as functions of $x$ so that the Stokes multipliers of (2.17) are independent of $x$. The original $x$-equation (2.18) describes the appropriate evolution of the solutions of (2.17) in $x$, and the Painleve equation (2.11) with $v=0$ is the integrability condition. In this way an isospectral deformation leads, for self-similar solutions, to monodromy-preserving deformations associated with the irregular singular point.

We also want to point out that whereas the self-similar solutions of integrable evolution equations appear to give solvable nonautonomous ordinary differential equations, there are many members of the latter class which do not result from self-similar limits of the former. (See Example 2, Appendix I.)

II. Correlation Functions of the Ising Model. A most remarkable occurrence of Painlevé transcendents was discovered by Barouch et al. [4] and was incorporated into a powerful general framework by Sato et al. [7]. The interest here is in computing the $k$-point correlation functions of the rectangular Ising model in the scaling limit. First we recall some terminology about the Ising model. A spin variable $\sigma_{m n}= \pm 1$ is attached to each point of an $M \times N$ lattice. The energy $E(\sigma)$ corresponding to a configuration $\sigma=\left\{\sigma_{m n}\right\}$ is

$$
E(\sigma)=-J_{1} \sum_{m=1}^{N} \sum_{n=1}^{N} \sigma_{m n} \sigma_{m+1, n}-J_{2} \sum_{m=1}^{M} \sum_{n=1}^{N} \sigma_{m n} \sigma_{m, n+1}
$$

(periodic boundary conditions). The partition function is

$$
Z_{M N}=\sum_{\sigma} e^{-\beta E(\sigma)}, \quad \beta=\frac{1}{k T},
$$

(summed over all possible $\sigma$ ), and the $k$-point correlation functions are

$$
\varrho_{k}\left(\left(m_{1}, n_{1}\right), \ldots,\left(m_{k}, n_{k}\right)\right)=Z_{M N}^{-1} \sum_{\sigma} \sigma_{m_{1} n_{1}} \ldots \sigma_{m_{k} n_{k}} e^{-\beta E(\sigma)} .
$$

One is interested in obtaining explicit formulae for these quantities as $M, N \rightarrow \infty$. It is well known that the partition function is not analytic at some critical $\beta_{c}$, corresponding to a critical temperature $T_{c}$. The scaling limit is a continuum limit of the lattice as $T \rightarrow T_{c}^{ \pm}$[13]. The 2-point correlation functions and their scaling limits were first evaluated and studied in [4]. Of particular interest for us is the fact that the 2-point functions in the scaling limit admit closed expressions in terms of solutions of the third Painleve equation (see the survey by Tracy [13]). Without invoking any deformation ideas, $\mathrm{Wu}$ et al. derived series expansions for the correlation functions; certain of these expansions for $k=2$ are equivalent to the Neumann series subsequently studied by Ablowitz et al. [12], and identical to iterative solutions obtained from our singular integral equations (see Sect. 4 below).

The deformation idea was introduced into this circle of problems by Sato et al. [7]. Their work presents a remarkable synthesis of apparently unrelated fields : the theory of rotations in Clifford algebras, monodromy-preserving deformations, and 
quantum field theory. Certain of their discoveries show a particularly strong similarity to facts familiar from inverse-scattering theory and lead directly into the investigations of the present paper.

We present a brief sketch of the basic deformation problem of [7] (algebraic details of the connection are given in Appendix III). [7] deals with solutions of the two-dimensional Dirac equation for $w=\left(\begin{array}{l}w_{+} \\ w_{-}\end{array}\right)$,

$$
\frac{\partial}{\partial z} w_{-}=m w_{+}, \quad \frac{\partial}{\partial \bar{z}} w_{+}=m w_{-}, \quad m>0
$$

which: are multivalued, having root type branch points at $2 n$ points $a_{1}, \ldots, a_{k}$, $\bar{a}_{1}, \ldots, \bar{a}_{k}$

$$
\begin{aligned}
& w_{ \pm}\left(a_{v}+e^{2 \pi i}\left(z-a_{v}\right), \bar{a}_{v}+e^{-2 \pi i}\left(\bar{z}-\bar{a}_{v}\right)\right) \\
& \quad=-e^{-2 \pi i l_{v}} w(z, \bar{z}),
\end{aligned}
$$

satisfy certain growth conditions at $\left(a_{v}, \bar{a}_{v}\right), v=1, \ldots, k$ and decay as $e^{-2 m|z|}$ as $|z| \rightarrow \infty$. For $n=1$, the only solution is a modified Bessel function of the second kind. In general, the space $W^{k}$ of such solutions is $k$ dimensional [7]. Let

$$
\mathbf{W}=\left(\begin{array}{c}
w^{(1)} \\
\vdots \\
w^{(k)}
\end{array}\right), \quad w^{(j)}=\left(w_{+}^{(j)}, w_{-}^{(j)}\right)^{T}
$$

be a basis (appropriately normalized) of $W^{k}$. There is a differential equation satisfied by $\mathbf{W}$,

$$
M_{F} \mathbf{W}=\left(B \frac{\partial}{\partial z}+\bar{B} \frac{\partial}{\partial \bar{z}}+E\right) \mathbf{W}
$$

where $M_{F}=z \frac{\partial}{\partial z}-\bar{z} \frac{\partial}{\partial z}+\frac{1}{2}\left(\begin{array}{rr}1 & 0 \\ 0 & -1\end{array}\right)$ and $B, \bar{B}, E$ are constant matrices which depend on $l_{v}, a_{v}$ and $\bar{a}_{v}, v=1, \ldots, k$. Equation (2.22) together with (2.19) completely characterizes the space $W^{k}$. One now asks: how do the matrices in (2.22) change as functions of the branch points $a_{j}, \bar{a}_{j}$ ? Because these matrices characterize $W^{k}$, which in turn is the space of solutions determined by the monodromy requirement (2.20), this question is about deformations of (2.19), (2.22) preserving the root monodromy and growth conditions. The solutions of the deformation equations lead to closed expressions for the $k$-point functions; for $k=2$, these reduce to the formulae mentioned above involving the third Painlevé transcendent. In [7], Eq. (3.3.39), Satō et al. point out that a formal Laplace transform

$$
\mathbf{W}(z, \bar{z})=\int \frac{d u}{2 \pi u}\left(\begin{array}{c}
\sqrt{u} \\
\sqrt{u^{-1}}
\end{array}\right) e^{m\left(z u+\bar{z} u^{-1}\right)} \hat{w}(u)
$$

converts (2.19), (2.22) into

$$
\left(u \frac{d}{d u}+m A u-G^{-1} m \bar{A} G u^{-1}+F\right) \hat{w}(u)=0 .
$$


This again produces a system of ordinary differential equations with polynomial coefficients, with irregular singular points at $u=0, \infty$. These equations involve the $a_{j}, \bar{a}_{j}$, and other parameters. We may ask: how should these other parameters change as functions of $a_{j}, \bar{a}_{j}$, if the Stokes multipliers of (2.23) are to remain constant? For $k=1$, the system of deformation equations is trivial, and for $k=2$, one recovers the sinh-Gordon similarity solution (i.e. a special case of Painlevé III). The exact connections are given in Appendices I and III.

\section{C. Classical Work}

The work of Satō et al. was in part stimulated by, and then re-applied to, some classical problems of deformation theory. We have already mentioned the work of Fuchs [9] on Painlevé VI as a deformation equation. The general system of deformation equations for (2.2) was derived by Schlesinger [14]:

$$
\begin{gathered}
\frac{\partial A^{j}}{\partial a_{i}}=\frac{\left[A^{i}, A^{j}\right]}{a_{j}-a_{i}}, \\
\sum_{j=1}^{n} \frac{\partial A^{j}}{\partial a_{i}}=0 .
\end{gathered}
$$

[7, Part II] details a constructive solution method for (2.24) based on a specialization of the deformation theory of the Dirac equation (Sect. B, above). Equation (2.24), of course, relates to monodromy groups of systems whose singular points are all regular. The question of irregular singular points was also taken up, by Garnier [15], but (as far as we can tell) on a purely formal level, by analogy with results of Fuchs on the regular-singular case. Garnier considers, in connection with Painlevé II, the 2nd order equation for $y(\zeta)$,

$$
y_{\zeta \zeta}=\left[\sum_{0}^{m} a_{j} \zeta^{j}+\sum_{0}^{n}\left(\frac{3 / 4}{\left(\zeta-\lambda_{k}\right)^{2}}+\frac{\varrho_{k}}{\zeta-\lambda_{k}}\right)\right] y .
$$

He then asks: how can one complement $(2.25)$ by an equation

$$
y_{x}=A y+B y_{\zeta}
$$

with $A, B$ rational in $\zeta$, so that $(2.25),(2.26)$ is an integrable system (in the sense: $\left.y_{x \zeta \zeta}=y_{\zeta \zeta x}\right)$ ? If $n=0$ in (2.25) and $\lambda_{0}=\lambda$, it turns out that one possible choice for (2.26) leads to Painlevé II as the integrability condition,

$$
\lambda^{\prime \prime}=2 \lambda^{3}+x \lambda-v .
$$

There has apparently been no discussion, so far, of the deformation theory underlying (2.25), (2.26), even though the work of Birkhoff $[16,17]$ presents most of the necessary ideas relating to the monodromy concept for an irregular singular point $^{1}$. Starting from the requirement that the Stokes multipliers of (2.25) [or rather, of (1.1)] be independent of $x$, we will prove (in Sect. 3) that an equation like (2.26), with rational $A, B$, must be satisfied.

1 After this work was completed, we learned of work of K. Ueno on this question (see Sect. 5) 
2D. Further Connections Between Isospectral and Monodromy-Preserving Deformations

Of the three deformation problems listed in Sect.2A, only two have been shown to be related up to this point of our discussion.

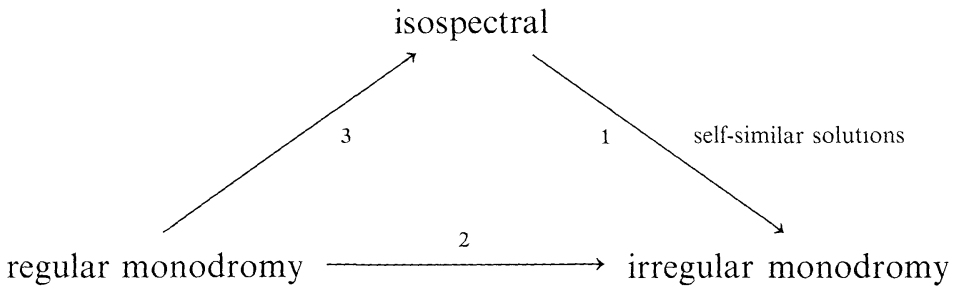

It is probably not surprising that connection 2 can be established. If one coalesces two or more regular singular points of an ordinary differential equation, one expects to get an irregular one. Indeed, Garnier [15] states (without details) that an equation such as (2.25) is obtained from one with all singular points regular by confluence, with the corresponding deformation equations passing into each other. This amounts to the observation of Painlevé (see, e.g. [1]) that the 6th equation, (2.4), (which is the integrability condition for a regular monodromy-preserving deformation) yields all other Painlevé equations I-V (associated with irregular monodromy) by appropriate limiting procedures. Yet, the geometric content of this statement is still very obscure.

Much more surprising is connection 3, again due in large part to Garnier [18]. It is fairly well known (Davis [19]) that the change of variables

$$
f=\sqrt{x} g, \quad t=\frac{2}{3} x^{3 / 2}
$$

converts Painlevé II into

$$
g^{\prime \prime}=2 g^{3}-2 g-\frac{1}{t} g^{\prime}+\frac{1}{9} \frac{g}{t^{2}}-\frac{2 v}{3 t}
$$

which, for $|t| \rightarrow \infty$ in certain regions of the $t$ plane reduces to

$$
g^{\prime \prime}=2 g^{3}-2 g \text {. }
$$

This is solvable in terms of elliptic functions. Indeed, the solutions of Painlevé II are "asymptotically elliptic" (much as Bessel functions are asymptotically trigonometric). Garnier takes the general Schlesinger system (2.24), lets the $a_{j} \rightarrow \infty$ so that $\frac{a_{i}}{a_{j}} \rightarrow \frac{\alpha_{i}}{\alpha_{j}}$ (fixed) and sets $\alpha_{j} \log \alpha_{j}=\tau_{j}$; then (2.24) formally becomes

$$
\begin{aligned}
& \frac{\partial A^{j}}{\partial \tau_{i}}=\frac{\left[A^{i}, A^{j}\right]}{\alpha_{j}-\alpha_{i}}, \\
& \sum_{j=1}^{n} \frac{\partial A^{j}}{\partial \tau_{i}}=0 .
\end{aligned}
$$


This is now an autonomous system, which Garnier integrates in terms of Abelian functions. It is remarkable that his basic lemma says that the eigenvalues of the matrix

$$
\prod_{i=1}^{n}\left(\zeta-\alpha_{i}\right) \sum_{j=1}^{n} \frac{A^{j}}{\zeta-\alpha_{j}}
$$

are unchanged when the $A^{j}$ solve (2.27) - a typical isospectral flow. One special case singled out (and solved) by Garnier is the system

$$
\begin{aligned}
& \xi_{i}^{\prime \prime}=-\lambda_{i} \xi_{i}+\left(2 \sum_{1}^{n} \xi_{i} \eta_{j}\right) \xi_{i} \\
& \eta_{i}^{\prime \prime}=-\lambda_{i} \eta_{i}+\left(2 \sum_{1}^{n} \xi_{j} \eta_{j}\right) \eta_{i}
\end{aligned}
$$

which is now known $[20,21]$ to contain the finite-gap $\mathrm{KdV}$ theory as special case. It was, in fact, discovered some 60 years earlier by C. Neumann as describing uncoupled harmonic oscillators constrained to move on a sphere.

Garnier's system (2.27) covers a large class of the integrable periodic problems solvable by inverse spectral methods. There are other known periodic isospectral flows contained in (2.27). We will discuss these in the next paper, in which we will also re-interpret Garnier's method in the language of isospectral flows.

\section{The General Solution of Painlevé II}

\section{A. Outline}

We have already mentioned (and full details are given in Appendix I) that $r=q_{x}$ and

$$
q_{x x}=2 q^{3}+x q-v
$$

are the integrability conditions of the Eqs. (3.2) and (3.3) below

$$
\begin{aligned}
& v_{1 \zeta}=\left(-4 i \zeta^{2}-i\left(x+2 q^{2}\right)\right) v_{1}+\left(4 \zeta q+\frac{v}{\zeta}+2 i r\right) v_{2}, \\
& v_{2 \zeta}=\left(4 \zeta q+\frac{v}{\zeta}-2 i r\right) v_{1}+\left(4 i \zeta^{2}+i\left(x+2 q^{2}\right)\right) v_{2}, \\
& v_{1 x}=-i \zeta v_{1}+q v_{2}, \\
& v_{2 x}=q v_{1}+i \zeta v_{2} .
\end{aligned}
$$

The method of isospectral deformation, or IST, concentrates its principal attention on (3.3). In order to implement the method, one must have some information on $q$ as function of $x$; for example, that it decays to zero or a constant as $x \rightarrow \pm \infty$ and also that certain moments exist. If

$$
\int_{-\infty}^{\infty}(1+|x|)|q| d x<\infty
$$


one can define the fundamental solution matrices $\hat{\Phi}(x, \zeta)$ and $\hat{\Psi}(x, \zeta)$ by the asymptotic properties

$$
\hat{\Phi}(x, \zeta) \rightarrow \hat{E}(x, \zeta)=\left(\begin{array}{cc}
e^{-i \zeta x} & 0 \\
0 & e^{i \zeta x}
\end{array}\right) \quad \text { as } \quad x \rightarrow-\infty
$$

and

$$
\hat{\Psi}(x, \zeta) \rightarrow \hat{E}(x, \zeta) \quad \text { as } \quad x \rightarrow+\infty
$$

Condition (3.4) ensures that certain analyticity properties hold, and in particular that the scattering matrix $A(\zeta)=\hat{\Phi}^{-1} \hat{\Psi}$ is defined with its diagonal entries admitting analytic extension. The $\zeta$ behavior of $A(\zeta)$ (or the $(\zeta, t)$ behavior of $A(\zeta, t)$ - see [33]) is inferred from (3.2)

For the class of equations and solutions we wish to discuss, condition (3.4) does not hold. Therefore, we propose a new method, in which one focuses central attention on Eq. (3.2) and uses (3.3) as an auxiliary equation. We note straightaway that (3.2) is much simpler: the coefficients are polynomials in the independent variable $\zeta$. The points $\zeta=0$ and $\zeta=\infty$ are regular and irregular singular points of the equation, respectively, and the solution matrix is a meromorphic function of $\zeta$ on an appropriate Riemann surface. If $v$ is an integer, the solution matrix is meromorphic in the finite complex $\zeta$ plane; otherwise, one must introduce the multisheeted Riemann surface of $\zeta^{\nu}$.

The steps in the method exactly parallel the steps used in IST. First, at a given value of $x$, where $q$ and $q_{x}$ are given, one determines various properties of the solution matrix connected with the singular points $\zeta=0$ and $\zeta=\infty$ of the equation. Around the singular point $\zeta=\infty$, one has the Stokes phenomenon: the analytic continuation of a solution from one sector to another does not have as its asymptotic expansion, as $\zeta \rightarrow \infty$ in the new sector, the analytic continuation of the asymptotic expansion in the first sector. If one identifies a solution matrix in each of the sectors abutting infinity by a fixed asymptotic behavior, then these solutions will not evolve from one sector to another in a continuous fashion, but will be connected by Stokes multiplier matrices. The entries of these Stokes multiplier matrices are the Stokes multipliers and are part of the characteristic data of the singular point. The other data needed at $\zeta=\infty$ are the coefficients of the polynomial in the exponent of the formal asymptotic expansion of the fundamental solution matrix. If the rank of the irregular singular point is $r$, then the components of the solution vector will have asymptotic expansions of the form

$$
\exp \left\{\sum_{i=1}^{r} \omega_{i} \zeta^{i}\right\} \zeta^{l}\left\{1+O\left(\frac{1}{\zeta}\right)\right\}
$$

In the case under discussion, $r=3, \omega_{3}=4 i / 3, \omega_{2}=0, \omega_{1}=i x, l=0$. One also needs to know how the solution changes as the point $\zeta=\infty$ is encircled; does it return to the value in the first sector? This can be determined by examining the behavior of the solution in the neighborhood of the regular singular point. Thus, one introduces the monodromy matrix at $\zeta=0$. Finally, one must specify the 
connection matrix between canonical fundamental solution matrices at $\zeta=0$ and $\zeta=\infty$. The characteristic parameters at $\zeta=\infty$ (the Stokes multiplier matrices and $\left.\left\{\omega_{j}\right\}, l\right)$, the monodromy matrix at $\zeta=0$, and the connection matrix are together called the transform data.

The second step in the method is crucial. We ask: how do the transform data evolve as the parameters $\omega_{i}$ in (3.6) change? From (3.3), we will show that their evolution is trivial: they are constant.

Therefore, the third step is to reconstruct the coefficients $r$ and $q$ in (3.2) and hence $q$ and $q_{x}$ at any $x$. This is achieved by deriving linear singular integral equations for the columns of the fundamental solution matrix.

We now discuss these steps in detail in connection with the system (3.1)-(3.3).

\section{B. The Direct Transform}

A formal asymptotic analysis of (3.2) at $\zeta=\infty$ will show that the two linearly independent solutions have the expansions

$$
\begin{aligned}
& \psi^{(1)}(\zeta, x) \sim \tilde{\psi}^{(1)}=e^{-4 i \zeta^{3} / 3-i \zeta x}\left(\begin{array}{l}
1-\frac{i}{2 \zeta}\left(q_{x}^{2}-x q^{2}-q^{4}+2 v q\right)+\ldots \\
\frac{i q}{2 \zeta}+\ldots
\end{array}\right), \\
& \psi^{(2)}(\zeta, x) \sim \tilde{\psi}^{(2)}=e^{4 i \zeta^{3} / 3+i \zeta x}\left(\begin{array}{c}
-\frac{i q}{2 \zeta}+\ldots \\
1+\frac{i}{2 \zeta}\left(q_{x}^{2}-x q^{2}-q^{4}+2 v q\right)+
\end{array}\right) .
\end{aligned}
$$

Since our concern is with (3.1), we will henceforth write $q_{x}$ for $r$. If (3.1) holds, $q_{x}^{2}-x q^{2}-q^{4}+2 v q=-\int^{x} q^{2} d x+$ const and if $q, q_{x}$ tend to zero sufficiently rapidly as $x \rightarrow \infty$, this coefficient is simply $\int_{x}^{\infty} q^{2} d x$. The growth or decay of the two formal asymptotic expansions $\tilde{\psi}^{(1)}(\zeta, x)$ and $\tilde{\psi}^{(2)}(\zeta, x)$ as $\zeta \rightarrow \infty$ is determined by the exponential factor $e^{ \pm 4 i \zeta^{3} / 3}$; the former (latter) series is dominant (recessive), meaning exponentially growing (decaying), as $\zeta \rightarrow \infty$ in the sectors $S_{1}, S_{3}$ and $S_{5}$ shown in Fig. 1 below, and recessive (dominant) in the sectors $S_{2}, S_{4}, S_{6}$, $S_{j}=\left\{\zeta|| \zeta \mid>\varrho\right.$, some $\left.\varrho, \frac{(j-1) \pi}{3} \leqq \arg \zeta<\frac{j \pi}{3}\right\}$.

The initial lines of the sectors $S_{j}$ are called the anti-Stokes lines. The lines on which the solutions are maximally dominant or recessive (in this case $\pi / 6+\pi j / 3$, $j=0,1, \ldots, 5)$ are called the Stokes lines.

Consider the solutions $\psi_{1}^{(1)}(\zeta, x)$ and $\psi_{1}^{(2)}(\zeta, x)$ of (3.2) which in $S_{1}$ have the asymptotic expansions $\tilde{\psi}^{(1)}$ and $\tilde{\psi}^{(2)}$. These solutions will usually be defined by integral representations following the procedure suggested by Birkhoff [16], or as solutions to integral equations. Then, by standard methods (steepest descent, iterative solutions), one can find asymptotic expansions for these solutions in other 


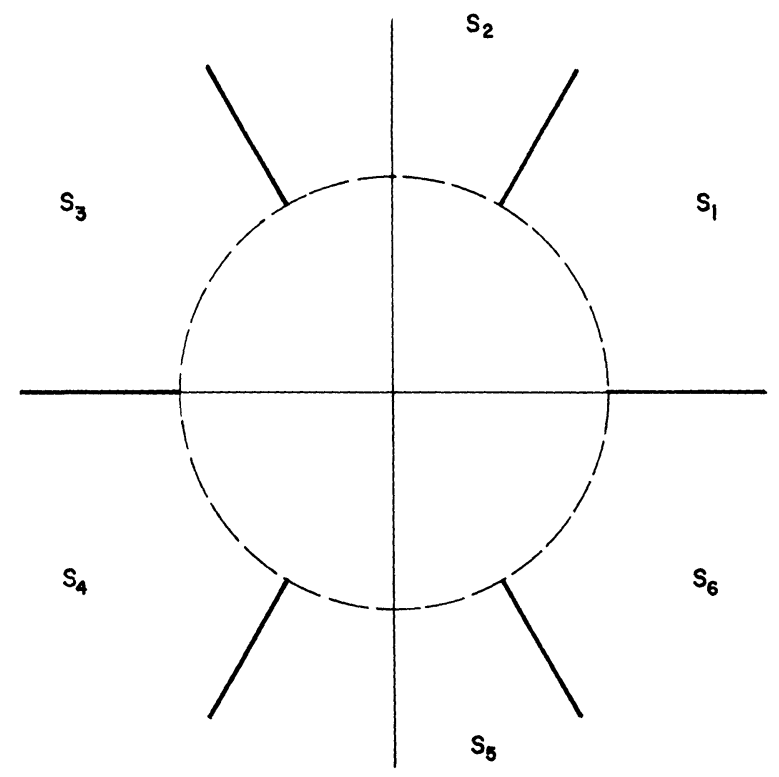

Fig. 1. The six sectors of infinity for (3.2)

sectors and, by taking appropriate linear combinations, one can determine canonical bases $\left\{\psi_{j}^{(1)}(\zeta, x), \psi_{j}^{(2)}(\zeta, x)\right\}_{j=2}^{7}$ which have the properties

$$
\psi_{j}^{(1)}(\zeta, x) \sim \tilde{\psi}^{(1)}(\zeta, x), \quad \psi_{j}^{(2)}(\zeta, x) \sim \tilde{\psi}^{(2)}(\zeta, x) \quad \text { in } S_{j} .
$$

We emphasize that the sector $S_{j}$ includes its initial ray on which the asymptotic expansions are neutral (neither growing nor decaying). The fundamental matrix $\Psi_{j}(\zeta, x)$ will not, in general, be equal to its contiguous neighbors $\Psi_{j-1}$ and $\Psi_{j+1}$, but will be related to them by the Stokes multiplier matrices $A_{j}(x)$,

$$
\Psi_{j+1}(\zeta, x)=\Psi_{j}(\zeta, x) A_{j}(x)
$$

Each $A_{j}(x)$ is triangular and has the form $\left(\begin{array}{cc}1 & a_{j} \\ 0 & 1\end{array}\right)$ or $\left(\begin{array}{cc}1 & 0 \\ a_{j} & 1\end{array}\right)$.

The entries $a_{j}$ are called the Stokes multipliers. The reason the matrices are triangular is this: one can show by analytic continuation of the integral representation, that a solution which is recessive in $S_{j}$ admits analytic continuation to $S_{j+1}$ and has the same asymptotic expansion (which is now dominant) there [16]. On the other hand, the dominant solution in a sector $S_{j}$ may need to pick up a recessive component before it can represent a recessive solution in the neighboring sector $S_{j+1}$.

This phenomenon was first discovered by Stokes in 1857 in his analysis of the Airy function

$$
\operatorname{Ai}(x)=\frac{1}{2 \pi} \int_{-\infty}^{\infty} e^{i k x+i k^{3} / 3} d k
$$


Stokes noticed that whereas the asymptotic expansion

$$
\operatorname{Ai}(x) \sim \frac{1}{2 \sqrt{\pi} x^{1 / 4}} \exp \left(-\frac{2}{3} x^{3 / 2}\right)
$$

is valid in the Poincaré sense in the region $|\arg x|<\pi$, it is necessary that a portion of the solution whose asymptotic behavior is $\frac{1}{2 \sqrt{\pi} x^{1 / 4}} \exp \left(\frac{2}{3} x^{3 / 2}\right)$ be added before $\arg x$ reaches $\pi$. At which value of $\arg x$ the extra portion is first added was the subject of debate, a debate only recently resolved by Olver [22], who pointed out that uniform bounds on the error were only obtainable for $|\arg x|<\frac{2 \pi}{3}$, that is, up to the last Stokes line before $\arg x=\pi$. In fact, Stokes himself knew by direct calculation that the asymptotic representation (3.10) was a poor approximation to the exact solution, which he computed by power series, once $|\arg x|$ exceeded $\frac{2 \pi}{3}$. For our purposes, it is not crucial to know on which line a given dominant solution must pick up a multiple of the recessive solution; all we use is that in each sector, $\Psi_{j} \sim \tilde{\Psi}=\left(\tilde{\psi}^{(1)}, \tilde{\psi}^{(2)}\right)$ in the Poincaré sense of asymptotic expansions (that is, exponentially small terms can be omitted).

The Stokes multiplier matrices $A_{j}$ have certain symmetry properties which follow from the symmetry properties of (3.2). If $\Psi(\zeta, x)$ is a solution of (3.2), so is $M \Psi(-\zeta, x)$, where $M=\left(\begin{array}{ll}0 & 1 \\ 1 & 0\end{array}\right)$. Thus,

$\psi_{4}^{(1)}(\zeta, x)=M \psi_{1}^{(2)}(-\zeta, x), \psi_{5}^{(2)}(\zeta, x)=M \psi_{2}^{(1)}(-\zeta, x), \psi_{6}^{(1)}(\zeta, x)=M \psi_{3}^{(2)}(-\zeta, x)$

as each of these solutions is recessive in the sector indicated by the subscript, and recessive solutions are uniquely determined by their asymptotic expansion. The dominant solutions satisfy the same symmetry properties:

$$
\begin{aligned}
& \psi_{4}^{(2)}(\zeta, x)=M \psi_{1}^{(1)}(-\zeta, x), \psi_{5}^{(1)}(\zeta, x)=M \psi_{2}^{(2)}(-\zeta, x), \\
& \psi_{6}^{(2)}(\zeta, x)=M \psi_{3}^{(1)}(-\zeta, x) .
\end{aligned}
$$

Indeed, since $M \psi_{1}^{(1)}(-\zeta, x)$ goes like $\left(\begin{array}{l}0 \\ 1\end{array}\right) \exp \left(4 i \zeta^{3} / 3+i \zeta x\right)$ on $\arg \zeta=\pi$ and is also a dominant solution in $S_{4}$, it is exactly $\psi_{4}^{(2)}(\zeta, \mathrm{x})$. The remaining relations of $(3.10 \mathrm{~b})$ follow similarly. If $q(x)$ is real, then if $\Psi(\zeta, x)$ is a solution so is $M \Psi^{*}\left(\zeta^{*}, x\right)$, which will imply further restrictions on the Stokes multipliers [see (3.26)].

We now write down the fundamental solution matrices $\Psi_{j}(\zeta, x)=\left(\psi_{j}^{(1)}(\zeta, x)\right.$, $\left.\psi_{j}^{(2)}(\zeta, x)\right)$ which have the asymptotic behavior

$$
\tilde{\Psi}=\left(\begin{array}{l}
1-\frac{i}{2 \zeta}\left(q_{x}^{2}-x q^{2}-q^{4}+2 v q\right)+\ldots, \frac{-i q}{2 \zeta}+\ldots \\
\frac{i q}{2 \zeta}+\ldots, 1+\frac{i}{2 \zeta}\left(q_{x}^{2}-x q^{2}-q^{4}+2 v q\right)+\ldots
\end{array}\right)\left(\begin{array}{cc}
e^{-\theta} & 0 \\
0 & e^{\theta}
\end{array}\right),
$$


where $\theta=4 i \zeta^{3} / 3+i \zeta x$. From now on, we drop the subscript on $\Psi_{1}$.

$$
\begin{aligned}
& 0 \leqq \arg \zeta<\frac{\pi}{3}, \quad \Psi_{1}(\zeta, x) \equiv \Psi(\zeta, x)=\left(\psi^{(1)}, \psi^{(2)}\right), \\
& \frac{\pi}{3} \leqq \arg \zeta<\frac{2 \pi}{3}, \Psi_{2}(\zeta, x)=\Psi(\zeta, x)\left(\begin{array}{ll}
1 & 0 \\
a & 1
\end{array}\right) \\
& \frac{2 \pi}{3} \leqq \arg \zeta<\pi, \quad \Psi_{3}(\zeta, x)=\Psi_{2}(\zeta, x)\left(\begin{array}{ll}
1 & b \\
0 & 1
\end{array}\right) \\
& \pi \leqq \arg \zeta<\frac{4 \pi}{3}, \Psi_{4}(\zeta, x)=\Psi_{3}(\zeta, x)\left(\begin{array}{ll}
1 & 0 \\
c & 1
\end{array}\right) \\
& \frac{4 \pi}{3} \leqq \arg \zeta<\frac{5 \pi}{3}, \Psi_{5}(\zeta, x)=\Psi_{4}(\zeta, x)\left(\begin{array}{ll}
1 & d \\
0 & 1
\end{array}\right) \\
& \frac{5 \pi}{3} \leqq \arg \zeta<2 \pi, \quad \Psi_{6}(\zeta, x)=\Psi_{5}(\zeta, x)\left(\begin{array}{ll}
1 & 0 \\
e & 1
\end{array}\right) \\
& 2 \pi \leqq \arg \zeta<\frac{7 \pi}{3}, \Psi_{7}(\zeta, x)=\Psi_{6}(\zeta, x)\left(\begin{array}{ll}
1 & f \\
0 & 1
\end{array}\right) .
\end{aligned}
$$

We remark now, and prove later, that each $\Psi_{j}(\zeta, x)$ also satisfies the $x$-equation (3.3). The fundamental matrix $\Psi_{7}(\zeta, x)$ is defined on a sector including the positive real axis $\arg \zeta=2 \pi$. If $\Psi$ is meromorphic in the complex $\zeta$-plane, $\Psi_{7}=\Psi$. However, multivaluedness cannot be seen from the asymptotic behavior at $\zeta=\infty$, but can be inferred from the behavior of the solution about $\zeta=0$. This we shall consider in a moment. First, however, let us use the symmetry properties (3.10) to show that $d=a, e=b, f=c$. For example, since $\psi_{2}^{(1)}=\psi_{1}^{(1)}+a \psi_{1}^{(2)}$, one has $M \psi_{2}^{(1)}(-\zeta)$ $=M \psi_{1}^{(1)}(-\zeta)+a M \psi_{1}^{(2)}(-\zeta)$ or $\psi_{5}^{(2)}(\zeta)=\psi_{4}^{(2)}(\zeta)+a \psi_{4}^{(1)}(\zeta)$. Comparison with (3.12e) shows $d=a$. The remaining relations follow similarly.

It is straightforward to write down linearly independent solutions of (3.2) near $\zeta=0$. When $v$ is not a half integer $\frac{2 n+1}{2}$, they are of the form

$$
\begin{aligned}
& \phi^{(1)}(\zeta, x)=\frac{1}{\sqrt{2} \zeta^{v}} e^{-u(x)}\left\{\left(\begin{array}{r}
1 \\
-1
\end{array}\right)-\frac{i \zeta}{1-2 v}\left(2 q_{x}+2 q^{2}+x\right)\left(\begin{array}{l}
1 \\
1
\end{array}\right)+\ldots\right\}, \\
& \phi^{(2)}(\zeta, x)=\frac{1}{\sqrt{2}} \zeta^{v} e^{u(x)}\left\{\left(\begin{array}{l}
1 \\
1
\end{array}\right)+\ldots\right\},
\end{aligned}
$$

where the normalizing factors $e^{ \pm u(x)}, u_{x}=q(x)$ have been introduced in order that (3.13) satisfy (3.3). The coefficient vectors in (3.13) can always be chosen to alternate between $\left(\begin{array}{l}1 \\ 1\end{array}\right)$ and $\left(\begin{array}{r}1 \\ -1\end{array}\right)$; this is automatic when $v \neq n$, but we impose this pattern also when $v=n$. The reader can readily verify that even though $v$ be an integer, so that the difference $2 v$ of the indicial roots $-v$ and $+v$ is an integer, no 
logarithm terms are needed. When $v=\frac{2 n+1}{2}$, there will, in general, be logarithm terms. The two linearly independent solutions will then be $\phi^{(2)}(\zeta, x)$ and

$$
\phi^{(1)}(\zeta, x)=-i j \phi^{(2)}(\zeta, x) \ln \zeta+\phi^{(1)}(\zeta, x),
$$

where $\phi^{(1) \prime}$ has the form of $\zeta^{-v}$ times a holomorphic function. In (3.14), $j$ is proportional to the coefficient of $\zeta^{2 v-1 / 2}$ in the series (3.13a). For example, when $v=\frac{1}{2}$

$$
j=2\left(q_{x}+q^{2}+\frac{x}{2}\right) e^{-2 u} .
$$

Note that the logarithms will disappear if $j=0$; when $v=\frac{1}{2}$ this implies

$$
q_{x}+q^{2}+\frac{x}{2}=0 .
$$

(3.16) defines a one-parameter family of solutions of (3.1) for which the second order Painlevé equation reduces to a first order equation (see $3 \mathrm{~F}$ ).

The solutions $\phi^{(1)}$ and $\phi^{(2)}$ satisfy the symmetry condition

$$
\begin{aligned}
& M \phi^{(1)}\left(\zeta e^{-i \pi}\right)=-e^{v \pi i} \phi^{(1)}(\zeta)-\pi j e^{-v \pi i} \phi^{(2)}(\zeta), \\
& M \phi^{(2)}\left(\zeta e^{-i \pi}\right)=e^{-v \pi i} \phi^{(2)}(\zeta) .
\end{aligned}
$$

Also, from (3.14), if $\Phi(\zeta, x)$ is the fundamental matrix $\left(\phi^{(1)}, \phi^{(2)}\right)$ in $0 \leqq \arg \zeta<2 \pi$, then

$$
\Phi\left(\zeta e^{2 \pi i}, x\right)=\Phi(\zeta, x) J
$$

is a fundamental solution matrix in the sector $(2 \pi, 4 \pi)$. The matrix $J$ is

$$
J=\left(\begin{array}{cc}
e^{-2 \pi i v} & 0 \\
2 \pi j e^{2 \pi i v} & e^{2 \pi i v}
\end{array}\right),
$$

where $j$ is only nonzero when $v$ is a half integer, in which case $e^{-2 \pi i v}=e^{2 \pi i v}=-1$. We remark now, and prove later, that $J$ is independent of $x$.

Finally, we specify the relation between $\Psi(\zeta, x)$ and $\Phi(\zeta, x)$ to be

$$
\Psi(\zeta, x)=\Phi(\zeta, x) A
$$

where

$$
A=\left(\begin{array}{ll}
\alpha & \beta \\
\gamma & \delta
\end{array}\right)
$$

and $\alpha \delta-\beta \gamma=1$ since $\operatorname{det} \Psi=\operatorname{det} \Phi=1$.

The set of data

$$
T=\left\{a, b, c, \alpha, \beta, \delta, \alpha \delta-\beta \gamma=1, v, j, \omega_{1}, \omega_{2}, \omega_{3}, l\right\}
$$

is the transform data; in our case, $\omega_{3}=4 i / 3, \omega_{2}=l=0, \omega_{1}=i x$. 


\section{C. Some Properties of the Transform Data.}

With the conventions adopted here, the transform data are not uniquely specified by (3.2), (3.3) because there are some ambiguities in the definitions (3.13), (3.14) of $\Phi$. First, we consider this situation when $2 v$ is not an integer. The exponent $\pm u(x)$ in (3.13) is determined only up to an arbitrary constant; a change in this constant amounts to the multiplication of $\phi^{(1)}$ by some $k$ and of $\phi^{(2)}$ by $k^{-1}$. Therefore, a one-parameter family of connection matrices $A$ is consistent with a normalization of the type (3.13). One could remedy this by imposing

$$
\Phi\left(x_{0}, \zeta\right)\left(\zeta^{v},\right) \zeta_{\zeta=0}=\frac{1}{\sqrt{2}}\left(\begin{array}{rr}
1 & 1 \\
-1 & 1
\end{array}\right)
$$

but as the ambiguity will cause no problems, we shall not insist on such a condition. When $v$ is an integer, another potential indeterminacy appears because any linear combination $\phi^{(1)}+k \phi^{(2)}$ will be of the form $\zeta^{-n} x$ holomorphic function.

This would, however, violate the $\left(\begin{array}{r}1 \\ \pm 1\end{array}\right)$ patterns imposed on the coefficients in (3.13), and hence the symmetry (3.17). When $v=\frac{2 n+1}{2}$ and $j \neq 0$, there is again one free scaling parameter, and $A$ and the jump $j$ are determined up to this parameter.

The only really singular situation arises when $v=\frac{2 n+1}{2}$ and $j=0$. Then the symmetry condition (3.17) does not distinguish between different linear combinations $\phi^{(1)}+k \phi^{(2)}$. This case is exceptional in many respects; to illustrate its peculiar features, we turn now to an analysis of various relations among the transform data.

Our attitude is always that $x$ and $v$ are given, and that $q$ and $r$ in (3.2) are to be found. If the transform data do indeed determine those two complex numbers, then all of $a, b, c, j, \alpha, \beta, \gamma, \delta$ should depend on only two quantities in the list. Of course, $\alpha \delta-\beta \gamma=1$, and there will always be one free parameter in $A$ and $j$ due to the scaling freedom just discussed. Modulo this indeterminacy, we have the following results :

(A) As long as $v \neq \frac{2 n+1}{2}$ or $v=\frac{2 n+1}{2}$ and $j \neq 0$, any two of the three Stokes multipliers determine all transform data.

(B) If $v=\frac{2 n+1}{2}$ and $j=0, \frac{\alpha}{\beta}$ or $\frac{\beta}{\alpha}$ determines all remaining transform data (the second determining constant in this case being $j$ ). In particular, $a=b=c=(-1)^{n+1} i$.

Remark. The inverse problem for the exceptional case (B) can be solved - all systems (3.2) with such transform data can be constructed explicitly.

We now prove assertions (A), (B). To this end, we derive two sets of relations among the transform data; the relations are consistent but carry slightly different information. 
First, we note that from (3.19a) and (3.18a)

$\Psi\left(\zeta e^{2 \pi i}\right)=\Phi(\zeta) J A$,

and thus

$$
\Psi_{7}\left(\zeta e^{2 \pi i)}\left\{\left(\begin{array}{ll}
1 & 0 \\
a & 1
\end{array}\right)\left(\begin{array}{ll}
1 & b \\
0 & 1
\end{array}\right)\left(\begin{array}{ll}
1 & 0 \\
c & 1
\end{array}\right)\left(\begin{array}{ll}
1 & a \\
0 & 1
\end{array}\right)\left(\begin{array}{ll}
1 & 0 \\
b & 1
\end{array}\right)\left(\begin{array}{ll}
1 & c \\
0 & 1
\end{array}\right)\right\}^{-1}=\Psi(\zeta) A^{-1} J A .\right.
$$

Now choose $\zeta \in S_{1}$ (whence $\zeta e^{2 \pi i} \in S_{7}$ ) and let $|\zeta| \rightarrow \infty$; both $\Psi_{7}\left(\zeta e^{2 \pi i}\right)$ and $\Psi(\zeta)$ then tend to the same asymptotic matrices. Hence,

$$
\left(\begin{array}{ll}
1 & 0 \\
a & 1
\end{array}\right)\left(\begin{array}{ll}
1 & b \\
0 & 1
\end{array}\right)\left(\begin{array}{ll}
1 & 0 \\
c & 1
\end{array}\right)\left(\begin{array}{ll}
1 & a \\
0 & \frac{1}{1}
\end{array}\right)\left(\begin{array}{ll}
1 & 0 \\
b & 1
\end{array}\right)\left(\begin{array}{ll}
1 & c \\
0 & 1
\end{array}\right)=A^{-1} J^{-1} A
$$

If we write

$$
G=\left(\begin{array}{ll}
1 & 0 \\
a & 1
\end{array}\right)\left(\begin{array}{ll}
1 & b \\
0 & 1
\end{array}\right)\left(\begin{array}{ll}
1 & 0 \\
c & 1
\end{array}\right)\left(\begin{array}{ll}
0 & 1 \\
1 & 0
\end{array}\right)
$$

then

$$
A G^{2}=J^{-1} A \text {. }
$$

(3.22) does not use the symmetry (3.17) and so misses some information. Thus, we derive a second set of relations. In (3.19a), set $\zeta=\hat{\zeta} e^{-i \pi}$, and apply $M=\left(\begin{array}{ll}0 & 1 \\ 1 & 0\end{array}\right)$. Use $M \psi^{(1)}\left(\hat{\zeta} e^{-i \pi}\right)=\psi_{4}^{(2)}(\hat{\zeta}), M \psi^{(2)}\left(\hat{\zeta} e^{-i \pi}\right)=\psi_{4}^{(1)}(\hat{\zeta})$ on $\Psi$ and (3.17) on $\Phi$ in the resulting expression. Finally, re-express $\psi_{4}^{(1)}, \psi_{4}^{(2)}, \phi^{(1)}$ and $\phi^{(2)}$ in terms of $\psi^{(1)}, \psi^{(2)}$ by (3.12) and (3.19a), and equate coefficients of $\psi^{(1)}, \psi^{(2)}$. The result is :

$$
\begin{aligned}
b & =-\alpha \delta e^{v \pi i}-\beta \gamma e^{-v \pi i}+\pi j \alpha \beta e^{-v \pi i} \\
1+a b & =2 \alpha \gamma \cos v \pi-\pi j \alpha^{2} e^{-v \pi i} \\
1+b c & =-2 \beta \delta \cos v \pi+\pi j \beta^{2} e^{-v \pi i} \\
a+c+a b c & =\beta \gamma e^{v \pi i}+\alpha \delta e^{-v \pi i}-\pi j \alpha \beta e^{-v \pi i} .
\end{aligned}
$$

From (3.23), we deduce immediately that,

$$
a+b+c+a b c=-2 i \sin v \pi,
$$

so that any two Stokes multipliers (and $v$ ) determine the third [(3.22) almost yields (3.24); the sign of the right-hand side is not determined]. (3.23) is not convenient if one wants, as we do, to express everything in terms of these two Stokes multipliers. (3.22), on the other hand, is linear in the entries of $A$. A tedious but straightforward computation shows the following.

When $2 v$ is not an integer, or when $v=\frac{2 n+1}{2}$ and $j \neq 0,(3.22)$ has rank 2 , so that $\alpha, \beta, \gamma, \delta, j$ are determined up to two arbitrary constants; one is fixed by $\alpha \delta-\beta \gamma=1$, the other reflects the scaling freedom in $\Phi$.

When $v=n,(3.22)$ reduces to $I=I$, while (3.23) yields expressions for $\alpha, \beta, \gamma, \delta$ in terms of two Stokes multipliers (again, up to the one free constant). 
When $v=\frac{2 n+1}{2}$ and $j=0,(3.22)$ reduces to $I=I$ again. (3.23) can be solved for $a, b, c$ to give

$$
a=b=c=(-1)^{n+1} i \text {. }
$$

This has used $\alpha \delta-\beta \gamma=1$, and there are no further constraints contained in (3.23). Thus, $A$ depends on three free parameters. Two are connected with the indeterminacy of the definition of $\Phi$, as explained above. The third parameter, which can be taken to be $\frac{\alpha}{\beta}$ or $\frac{\beta}{\alpha}$ ( $\alpha$ or $\beta$ may be zero), labels different systems (3.2) corresponding to the one parameter family of solutions of (3.16) (this will be shown later in this section; the systems will be constructed explicitly).

Remark 1. (3.22) provides a quick proof of the absence of logarithms when $v=n$. Indeed, (3.22) says trace $G^{2}=\operatorname{trace} J^{-1}=2$; computing trace $G^{2}$, one finds that this implies $a+b+c+a b c=0$. The characteristic polynomial of $G$ then turns out to be $\lambda^{2}-1=0$, so its eigenvalues are \pm 1 . $G$ is therefore diagonalizable, and hence so is $G^{2}$. But $J=A G^{2} A^{-1}$ has Jordan block form unless $j=0$.

Remark 2. When can all Stokes multipliers be zero? Since then $G^{2}=I,(3.22)$ shows that $J=I$, whence $v=n$. This is another case of which all systems (3.2) can be constructed explicitly.

Remark 3. If, contrary to our previous position, $v$ is considered unknown, another parameter from the transform data must, of course, take it place. Suppose, for example, that $a, b, c$ are given. Then

$$
a+b+c+a b c=-2 i \sin v \pi
$$

determines $v \bmod 2$, and it is clear that (3.22) and (3.23) are not affected by a replacement $v \rightarrow v+2 m$. If $a, b, c$ are replaced by their negatives, (3.22) and (3.23) remain consistent provided $v \rightarrow v+2 m+1$. In other words, sets of transform data which differ only by an integer translation in $v$ and sign of $a, b, c$ are possible; this circumstance is related to Airault's [3] Bäcklund transformation, which produces a solution of (3.1) for $v \pm 1$ from a solution of (3.1) for $v$.

Remark 4. If $x, q, r$ in (3.2) are real, the symmetry $V(\zeta, x) \rightarrow M V^{*}\left(\zeta^{*}, x\right)$ shows

$$
a=-c^{*} \text {. }
$$

\section{D. The $x$-Dependence of $T$}

It has been mentioned repeatedly that the transform data are independent of $x$, provided that the Painleve equation (3.1) is satisfied. We verify this result, and also prove a strong converse: given matrix functions $\Psi_{j}(x, \zeta), \Phi(x, \zeta)$ with global connection properties characterized by transform data independent of $x$, there are unique systems (3.2), (3.3) satisfied by $\Psi_{j}, \Phi$. As a corollary, one can see that there is at most one set of functions $\Psi_{j}, \Phi$ possessing given transform data.

Because Eq. (3.2) is determined solely by the global connection properties of its solution, while (3.3) follows from the $x$-independence of the connection parameters, the whole theory can be rephrased in a much more geometrical manner without reference to differential equations; this is done in Sect. 6. 
In the remainder of this section, we deal with functions satisfying the following conditions :

(a) matrix functions $\Psi_{j}$ holomorphic in $S_{j}=\left\{\zeta|| \zeta \mid>0,(j-1) \frac{\pi}{3} \leqq \arg \zeta<\frac{j \pi}{3}\right\}$, such that

$$
\Psi_{j} \sim \tilde{\Psi}=\left(I+\frac{C_{1}}{\zeta}+\ldots\right)\left(\begin{array}{cc}
e^{-\theta} & 0 \\
0 & e^{\theta}
\end{array}\right), \quad \theta=\frac{4}{3} i \zeta^{3}+i \zeta x,
$$

as $|\zeta| \rightarrow \infty$ in $S_{j}$, and

$$
\Psi_{j+1}=\Psi_{j} A_{j}, \quad|\zeta|>\text { some } \varrho .
$$

(b) A matrix function $\Phi$ of the form

$$
\Phi(\zeta)=\hat{\Phi}(\zeta)\left(\begin{array}{cc}
\zeta^{-v} & 0 \\
0 & \zeta^{v}
\end{array}\right),
$$

with $\hat{\Phi}(\zeta)$ holomorphic, such that for $\zeta \in S_{1}$

$$
\Psi_{1}(\zeta)=\Phi(\zeta) A, \operatorname{det} A=1
$$

[For sake of simplicity, we omit the modifications necessary when $\Phi(\zeta)$ contains logarithms.]

The formal series $\tilde{\Psi}(\zeta)$ is assumed to have the symmetry

(c) $M \tilde{\Psi}(-\zeta) M=\tilde{\Psi}(\zeta), \quad M=\left(\begin{array}{ll}0 & 1 \\ 1 & 0\end{array}\right)$.

The functions studied earlier have all these properties.

We now prove the following facts:

Proposition 1. Let $\Psi_{j}$, $\Phi$ satisfy (3.2), with $\Psi_{j}$, $\Phi$ normalized as in (3.6) and (3.13). If the Painleve equation (3.1) holds (so that (3.2), (3.3) are compatible), then these $\Psi_{j}, \Phi$ are also solutions of the $x$-equation (3.3).

Proposition 2. If $\Psi_{j}$, $\Phi$ satisfy both (3.2) and (3.3), then the Stokes multipliers $A_{j}$, the connection matrix $A$, and the jump matrix $J$ are independent of $x$.

Remark. Propositions 1 and 2 show that the transform data introduced above are all independent of $x$.

Proposition 3. Let $\Psi_{j}, \Phi$ have properties $(a),(b),(c)$, and suppose that $A_{j}$ and $A$ are independent of $x$. Then $\Psi_{j}$, $\Phi$ satisfy differential equations of the form (3.2), (3.3).

Proposition 4. There can be no more than one set of functions $\Psi_{j}$, $\Phi$, satisfying properties $(a),(b),(c)$ above.

Proof of Proposition 1. Write Eq. (3.2) as $\Psi_{\zeta}=P \Psi$ and (3.3) as $\Psi_{x}=\mathrm{Q} \Psi$. Differentiate the first of these equations with respect to $x$ and solve the resulting inhomogeneous equations by variation of parameters to obtain

$$
\Psi_{x}=\Psi\left(\Psi^{-1} \Psi_{x}\right)_{\zeta_{0}}+\Psi \int_{\zeta_{0}}^{\zeta} \Psi^{-1} P_{x} \Psi d \zeta
$$


But the compatibility condition of (3.2) and (3.3) is $P_{x}=Q_{\zeta}+[Q, P]$ and, since $\Psi^{-1}\left(Q_{\zeta}+[Q, P]\right)=\frac{d}{d \zeta}\left(\Psi^{-1} Q \Psi\right)$, we have from (3.27) that

$$
\Psi_{x}=Q \Psi+\Psi\left[\Psi^{-1}\left(\Psi_{x}-Q \Psi\right)\right]_{\zeta_{0}} .
$$

Now let $\zeta_{0}$ tend to infinity on the initial ray of that sector in which $\Psi \sim \tilde{\Psi}$ as given in (3.6). One finds $\Psi_{x}-Q \Psi=O\left(\frac{1}{\zeta_{0}}\right)$, which tends to zero. Hence, $\Psi_{x}=Q \Psi$. A similar proof holds for $\Phi$.

Proof of Proposition 2. We prove that $A_{j}$ is independent of $x . A_{j}=\Psi_{j}^{-1} \Psi_{j+1}$, and thus $A_{j x}=-\Psi_{j}^{-1} \Psi_{j x} \Psi_{j}^{-1} \Psi_{j+1}+\Psi_{j}^{-1} \Psi_{j+1}=-\Psi_{j}^{-1} Q \Psi_{j+1}+\Psi_{j}^{-1} Q \Psi_{j+1}=0$. The proof that $A$ and $J$ are independent of $x$ is similar.

Remark. From the form of $J$ when, for example, $v=\frac{1}{2}$, it is by no means obvious that $j_{x}=0$. However, note that the $x$ derivative of $j=2\left(q_{x}+q^{2}+\frac{x}{2}\right) e^{-2 u}$ is zero by virtue of (3.1).

Proof of Proposition 3. First we note that (c) implies the following symmetry for the coefficient $C_{1}$ in the expansion $\tilde{\Psi}$ :

$-M C_{1} M=C_{1}$.

It follows that $C_{1,12}=-C_{1,21}$ and $C_{1,11}=-C_{1,22}$; we set $C_{1,12}=-\frac{i}{2} q$, and $C_{1,11}=p$. Now differentiate $\Psi_{j+1}=\Psi_{j} A_{j}$ with respect to $x$, and multiply by $\Psi_{j+1}^{-1}$ :

$$
\Psi_{j+1 x} \Psi_{j+1}^{-1}=\Psi_{j x} \mathrm{~A}_{j} \Psi_{j+1}^{-1}=\Psi_{j x} A_{j}\left(\Psi_{j} A_{j}\right)^{-1}=\Psi_{j x} \Psi_{j}^{-1} .
$$

$\Psi_{j x} \Psi_{j}^{-1}$ is therefore well-defined and holomorphic in a deleted neighborhood of $\infty$, and its asymptotic expansion is $\tilde{\Psi}_{x} \tilde{\Psi}^{-1}$, uniformly for $|\zeta|>$ some $\varrho$.

The asymptotic expansion is therefore convergent; we set

$$
\tilde{\Psi}_{x} \tilde{\Psi}^{-1}=Q(\zeta)
$$

$Q(\zeta)$ being a Laurent series in $\zeta^{-1}$, with a simple pole at $\zeta=\infty$. Near $\zeta=0$, we find in a similar way that

$$
\Phi_{x} \Phi^{-1}=\hat{\Phi}_{x} \hat{\Phi}^{-1}=\Psi_{1 x} \Psi_{1}^{-1} \equiv Q(\zeta) .
$$

But $\hat{\Phi}_{x} \hat{\Phi}^{-1}$ is holomorphic at $\zeta=0$, so that $Q(\zeta)$ contains no negative powers of $\zeta$. The explicit form of $Q(\zeta)$ is now easy to obtain by inserting the expansion $\tilde{\Psi}$ into (3.28). The result is

$$
Q(\zeta)=i \zeta\left(\begin{array}{rr}
-1 & 0 \\
0 & 1
\end{array}\right)+i\left[C_{1},\left(\begin{array}{rr}
-1 & 0 \\
0 & 1
\end{array}\right)\right]
$$

or

$$
Q(\zeta)=\left(\begin{array}{rr}
-i \zeta & q \\
q & i \zeta
\end{array}\right)
$$


Hence, $\Psi_{j}$, $\Phi$ satisfy an equation of the form (3.3) in $x$. A similar argument shows that $\Psi_{j \xi} \Psi_{j}^{-1}$ is well-defined and holomorphic away from $0, \infty$, so that

$$
\Psi_{j \zeta} \Psi_{j}^{-1}=\tilde{\Psi}_{\zeta} \tilde{\Psi}^{-1} \equiv P(\zeta) .
$$

$P(\zeta)$ has a double pole at $\zeta=\infty$. Near $\zeta=0$, we find

$$
P(\zeta)=\hat{\Phi}_{\zeta} \hat{\Phi}^{-1}+\frac{v}{\zeta} \hat{\Phi}\left(\begin{array}{rr}
-1 & 0 \\
0 & 1
\end{array}\right) \hat{\Phi}^{-1}
$$

Hence, $P(\zeta)$ contains at most the negative power $\zeta^{-1}$. We wish to show that in fact $\zeta^{-1}$ enters $P(\zeta)$ in the form

$$
\pm \frac{v}{\zeta}\left(\begin{array}{ll}
0 & 1 \\
1 & 0
\end{array}\right)
$$

Put $\hat{\Phi}(0)\left(\begin{array}{rr}-1 & 0 \\ 0 & 1\end{array}\right) \hat{\Phi}^{-1}(0)=\left(\varepsilon_{i j}\right)$. First, we note that the diagonal entries $\varepsilon_{11}, \varepsilon_{22}$ must be zero; otherwise there would be a term $\zeta^{ \pm v}$ in the expansion $\tilde{\Psi}$ [16]. In terms of the entries $\hat{\Phi}_{i j}(0)$, this means

$$
\hat{\Phi}_{11}(0) \hat{\Phi}_{22}(0)+\hat{\Phi}_{12}(0) \hat{\Phi}_{21}(0)=0 \text {. }
$$

But also $\operatorname{det} \hat{\Phi}(0)=1$, and a short computation shows that $\varepsilon_{12}=\varepsilon_{21}^{-1}$. Property (c) implies the symmetry

$$
P(-\zeta)=-M P(\zeta) M
$$

and this forces $\varepsilon_{12}=\varepsilon_{21}$, so $\varepsilon_{12}=\varepsilon_{21}= \pm 1$, as was to be shown.

It now remains to compute $\tilde{\Psi}_{\zeta} \tilde{\Psi}^{-1}=P(\zeta)$ through the $\zeta^{0}$ term. There are certain non-obvious cancellations, e.g. of $\zeta^{-2}$ terms, which are automatic by the analysis at $\zeta=0$. By (c), $C_{2}$ has the form $\left(\begin{array}{ll}y & z \\ z & y\end{array}\right)$. One finds that $\left[\right.$ with $\left.N=\left(\begin{array}{rr}-1 & \\ & 1\end{array}\right)\right]$

$$
P(\zeta)=\left(4 i \zeta^{2}+i x\right)\left\{N+\frac{1}{\zeta}\left[C_{1}, N\right]+\frac{1}{\zeta^{2}}\left(\left[C_{2}, N\right]+\left[N, C_{1}\right] C_{1}\right)\right\}
$$

or

$$
\begin{aligned}
P(\zeta)= & \left(4 i \zeta^{2}+i x+2 i q^{2}\right)\left(\begin{array}{rr}
-1 & 0 \\
0 & 1
\end{array}\right)+4 \zeta q\left(\begin{array}{ll}
0 & 1 \\
1 & 0
\end{array}\right) \\
& +(4 z-2 i p q)\left(\begin{array}{rr}
0 & 2 i \\
-2 i & 0
\end{array}\right) \pm \frac{\nu}{\zeta}\left(\begin{array}{ll}
0 & 1 \\
1 & 0
\end{array}\right) .
\end{aligned}
$$

If $r \equiv 4 z-2 i p q$, this is precisely the form required by (3.2), up to the ambiquity in the sign of $v$. The hypotheses do not allow one to distinguish between

$$
+\frac{v}{\zeta}\left(\begin{array}{ll}
0 & 1 \\
1 & 0
\end{array}\right), \quad \text { with } \Phi \text { as defined in (3.13), }
$$

and

$$
+\frac{(-v)}{\zeta}\left(\begin{array}{ll}
0 & 1 \\
1 & 0
\end{array}\right), \text { with } \Phi=\left(\phi^{(2)},-\phi^{(1)}\right) .
$$


Had we assumed, in addition, that $\hat{\Phi}(0)$ is proportional to $\left(\begin{array}{rr}1 & 1 \\ -1 & 1\end{array}\right)$, the choice $+\frac{v}{\zeta}\left(\begin{array}{ll}0 & 1 \\ 1 & 0\end{array}\right)$ would have been the only acceptable one.

Remark. In the first case, $q$ will satisfy Eq. (3.1). In the second case, $q$ solves $q_{x x}=2 q^{3}+x q+v$, but $-q$ again solves (3.1).

Proof of Proposition 4. Suppose that $\Psi_{j}$ and $\Psi_{j}^{\prime}(j=1, \ldots, 7)$ have property (a), with the same Stokes multipliers $A_{j}$, and suppose that $\Psi_{1}=\Phi A, \Psi_{1}^{\prime}=\Phi^{\prime} A$ with $\Phi, \Phi^{\prime}$ satisfying (b). Then

$$
\Psi_{j+1}^{\prime} \Psi_{j+1}^{-1}=\Psi_{j}^{\prime} A_{j} A_{j}^{-1} \Psi_{j}^{-1}=\Psi_{j}^{\prime} \Psi_{j}^{-1},
$$

so $S \equiv \Psi_{j}^{\prime} \Psi_{j}^{-1}$ is well-defined and holomorphic about $\zeta=\infty$, and from the asymptotic expansion one sees that $S$ is a Laurent series in $\zeta, S(\zeta)=I+O\left(\frac{1}{\zeta}\right)$. Near $\zeta=0$

$$
S(\zeta)=\Psi_{1}^{\prime} \Psi_{1}^{-1}=\Phi^{\prime} \Phi^{-1}=\hat{\Phi}^{\prime} \hat{\Phi}^{-1}=\mathrm{const}+O(\zeta),
$$

a Taylor series with no negative powers. Hence $S(\zeta) \equiv I$, as was to be shown.

\section{E. The Inverse Transform}

We now turn to the existence problem; are there solutions $\Psi_{j}$, $\Phi$ giving rise to a prescribed set of transform data? Here we derive linear singular integral equations

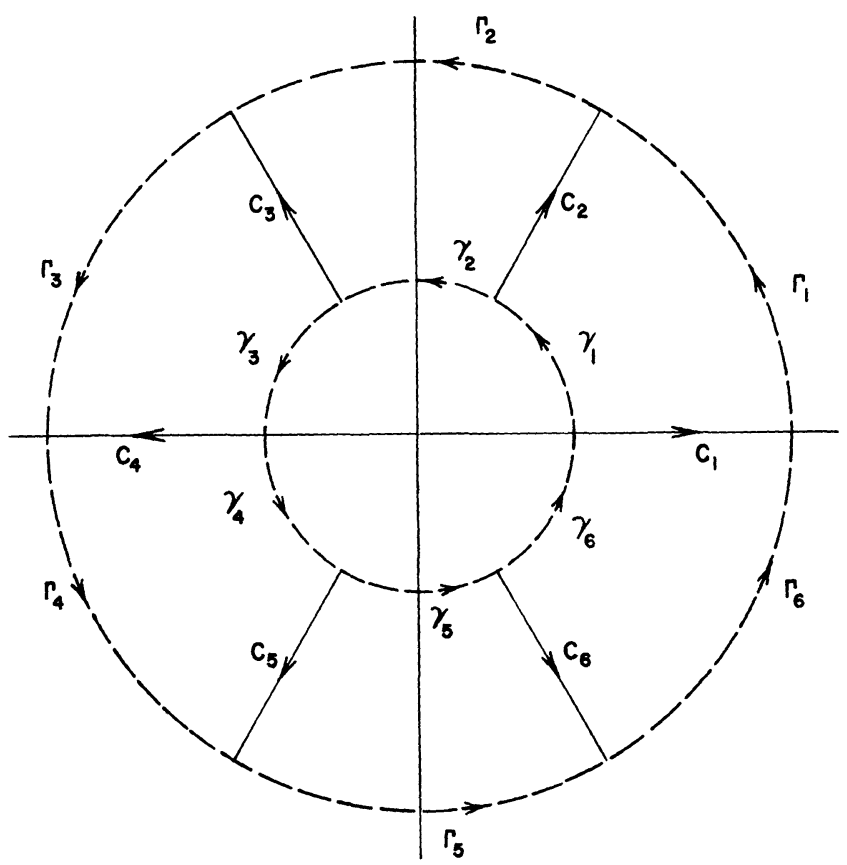

Fig. 2. The contours in the $\zeta$-plane for the inverse problem for Painlevé II 
from which the solutions $\Psi_{j}$ and $\Phi$ may be constructed. The parameters in these equations are $x$ and the Stokes multipliers. For simplicity of presentation, we take $v=n$, a positive integer. The results for $v \neq n$ are given in Appendix IV.

Consider

$$
\int_{C_{1}} \frac{\psi^{(1)}(\xi, x) e^{+\theta}}{\xi-\zeta} d \xi, \quad \theta=4 i \zeta^{3} / 3+i \zeta x
$$

for convenience, we take $\zeta$ to lie in the interior of $S_{1}$. Our goal is to write a system of linear singular equations for $\psi^{(1)}$ and $\psi^{(2)}$. The strategy will be to relate, by Cauchy's theorem, the integral along $C_{1}$ to one along $C_{2}$ and to continue this process around the singular point $\zeta=\infty$.

We begin by noticing that

$$
\int_{C_{1}} \frac{\psi^{(1)} e^{+\theta}}{\xi-\zeta} d \xi=2 \pi i \psi^{(1)}(\zeta) e^{\theta}-\frac{\pi i}{3}\left(\begin{array}{l}
1 \\
0
\end{array}\right)+\int_{C_{2}} \frac{\psi_{2}^{(1)}-a \psi_{2}^{(2)}}{\xi-\zeta} e^{\theta} d \xi+\int_{\gamma_{1}} \frac{\psi^{(1)} e^{\theta}}{\zeta-\zeta} d \xi,
$$

where we have used (3.12b). Now $\psi_{2}^{(2)} e^{\theta} \rightarrow \infty$ as $\zeta \rightarrow \infty$ in $S_{2}$, and therefore it is not possible to relate the term $-a \int_{C_{2}} \frac{\psi_{2}^{(2)} e^{\theta}}{\xi-\zeta} d \xi$ by contour integration to an integral along $C_{3}$. This integral is not transformed any further and appears in this form in the final equation. We can, however, continue with

$$
\int_{C_{2}} \frac{\psi_{2}^{(1)} e^{\theta}}{\xi-\zeta} d \xi=-\frac{\pi i}{3}\left(\begin{array}{l}
1 \\
0
\end{array}\right)+\int_{C_{3}} \frac{\psi_{3}^{(1)} e^{\theta}}{\xi-\zeta} d \xi+\int_{\gamma_{2}} \frac{\psi_{2}^{(1)} e^{\theta}}{\xi-\zeta} d \xi .
$$

Continuing in this manner around the $\zeta$-plane we find,

$$
\begin{aligned}
& \int_{C_{3}} \frac{\psi_{3}^{(1)} e^{\theta}}{\xi-\zeta} d \xi=-\frac{\pi i}{3}\left(\begin{array}{l}
1 \\
0
\end{array}\right)+\int_{C_{4}} \frac{\psi_{4}^{(1)}-c \psi_{4}^{(2)}}{\xi-\zeta} e^{\theta} d \xi+\int_{\gamma_{3}} \frac{\psi_{3}^{(1)} e^{\theta}}{\zeta-\zeta} d \xi, \\
& \int_{C_{4}} \frac{\psi_{4}^{(1)} e^{\theta}}{\xi-\zeta} d \xi=-\frac{\pi i}{3}\left(\begin{array}{l}
1 \\
0
\end{array}\right)+\int_{C_{5}} \frac{\psi_{5}^{(1)} e^{\theta}}{\xi-\zeta} d \xi+\int_{\gamma_{4}} \frac{\psi_{4}^{(1)} e^{\theta}}{\xi-\zeta} d \xi, \\
& \int_{C_{5}} \frac{\psi_{5}^{(1)} e^{\theta}}{\xi-\zeta} d \xi=-\frac{\pi i}{3}\left(\begin{array}{l}
1 \\
0
\end{array}\right)+\int_{C_{6}} \frac{\psi_{6}^{(1)}-b \psi_{6}^{(2)}}{\xi-\zeta} e^{\theta} d \xi+\int_{\gamma_{5}} \frac{\psi_{5}^{(1)} e^{\theta}}{\xi-\zeta} d \xi,
\end{aligned}
$$

and

$$
\int_{C_{6}} \frac{\psi_{6}^{(1)} e^{\theta}}{\xi-\zeta} d \xi=-\frac{\pi i}{3}\left(\begin{array}{l}
1 \\
0
\end{array}\right)+\int_{C_{1}} \frac{\psi^{(1)} e^{\theta}}{\xi-\zeta}+\int_{\gamma_{6}} \frac{\psi_{6}^{(1)} e^{\theta}}{\xi-\zeta} d \xi
$$

Here, we have used $\psi_{7}^{(1)}=\psi^{(1)}$, which is a consequence of the assumption $v=n$. Adding the Eqs. (3.32a) through (3.32f), we find

$$
\begin{aligned}
\psi^{(1)}(\zeta) e^{\theta(\zeta)}= & \left(\begin{array}{l}
1 \\
0
\end{array}\right)-\operatorname{Res}_{\xi=0} \frac{\psi^{(1)} e^{\theta(\xi)}}{\xi-\zeta}+\frac{a}{2 \pi i} \int_{C_{42}} \frac{\psi^{(2)} e^{\theta}}{\xi-\zeta} d \xi \\
& +\frac{b}{2 \pi i} \int_{C_{46}} \frac{\psi^{(2)} e^{\theta}}{\xi-\zeta} d \xi+\frac{b c}{2 \pi i} \int_{C_{64}} \frac{\psi^{(1)} e^{\theta}}{\xi-\zeta} d \xi,
\end{aligned}
$$


where we have substituted for $\psi_{j}^{(2)}, j=2,4,6$ from (3.12) in terms of $\psi^{(1)}, \psi^{(2)}$ and used identity (3.24), $a+b+c+a b c=0$. The contour $C_{42}$ is one which runs inward along $C_{4}$, then clockwise along $\gamma_{4}$ and $\gamma_{3}$ and goes out along $C_{2}$. The contours $C_{64}$ and $C_{46}$ (which is $-C_{64}$ ) are defined in a similar way (see Fig. 3).

Fig. 3. The contours $C_{42}$ and $C_{46}$

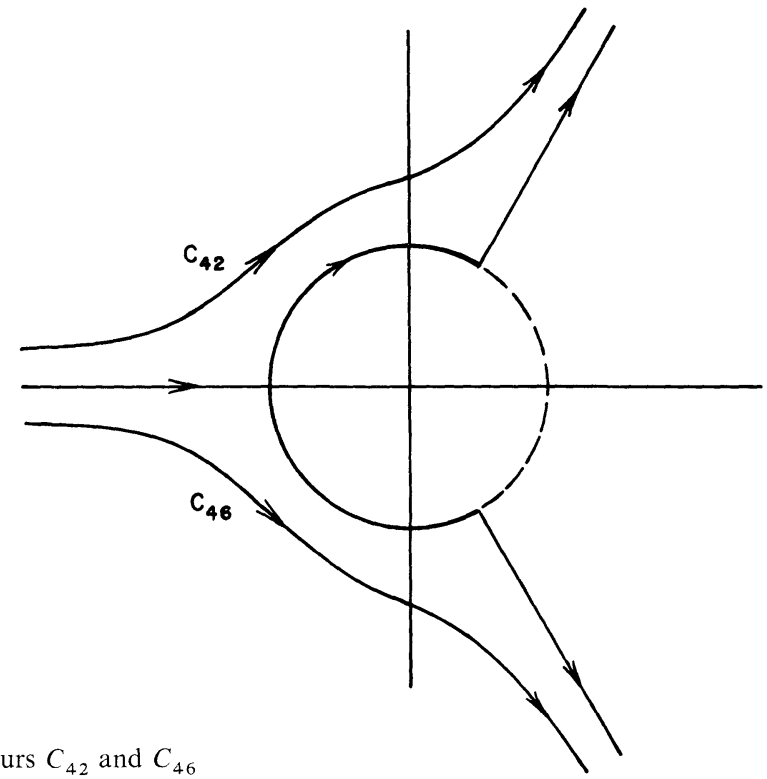

Remark. Note that the same contours are used in the integral representations of the Airy functions. In fact, (3.33) contains the Airy function representations as a limiting case [see example (i) which follows in $3 \mathrm{~F}$ ].

By considering

$$
\int_{C_{1}} \frac{\psi^{(2)} e^{-\theta}}{\xi-\zeta} d \xi,
$$

we find

$$
\begin{aligned}
\psi^{(2)} e^{-\theta}= & \left(\begin{array}{l}
0 \\
1
\end{array}\right)-\operatorname{Res}_{\xi=0} \frac{\psi^{(2)} e^{-\theta}}{\xi-\zeta}+\frac{b}{2 \pi i} \int_{C_{53}} \frac{\psi^{(1)} e^{-\theta}}{\xi-\zeta} d \xi+\frac{c}{2 \pi i} \int_{C_{51}} \frac{\psi^{(1)} e^{-\theta}}{\xi-\zeta} d \xi \\
& +\frac{a b}{2 \pi i} \int_{C_{53}} \frac{\psi^{(2)} e^{-\theta}}{\xi-\zeta} d \xi .
\end{aligned}
$$

Equations (3.33) and (3.35) are linear integral equations whose solution determines $\psi^{(1)}$ and $\psi^{(2)}$, and hence all the coefficients in the Eq. (3.2). In particular, from the asymptotic expansions (3.6), we known that

$$
\begin{aligned}
q & =\lim _{\zeta \rightarrow \infty} 2 i \zeta \psi_{, 1}^{(2)} e^{-\theta} \\
& =-\lim _{\zeta \rightarrow \infty} 2 i \zeta \psi_{, 2}^{(1)} e^{\theta}
\end{aligned}
$$

where the subscripts in (3.36) refer to the component, and not to the sector. 
The integral Eqs. (3.33) and (3.35) have been derived on the assumption that functions $\Psi_{j}$ with the prescribed Stokes multipliers exist. Conversely, one wants to answer the following questions:

(1) For which $x, a, b, n$ does a solution of (3.33), (3.35) exist?

(2) Does it exhibit the prescribed Stokes jumps?

(3) What properties of $q(x)$ can one deduce from the solution $\Psi$ so constructed?

(4) What is the dependence of $q(x)$ on the parameter $a, b$ ?

Some preliminary observations follow from the known properties of the Painlevé transcendent, $q(x)$. It is never an entire function of $x$, unless it is identically zero; it is, however, the ratio of two entire functions. This suggests that for given $n, a, b$, the solution of (3.33) and (3.35) will exist for all but a countable set of $x$. In particular, the inverse problem (Riemann-Hilbert problem) is not always solvable subject to the symmetry imposed on (3.2).

It is not too hard to see that any solution of the integral equations will have the required jumps; this follows from the behavior of the Cauchy integrals when $\zeta$ crosses an integration contour. The first problem, however, is existence of a solution. We have not found a proof; indeed, we have not really looked for one. The reason is this: other irregular-singular monodromy problems will lead to different, and more complicated sets of singular integral equations - see Sect. 4, for example. One needs a quite general theorem, if case-by-case existence proofs are to be avoided. It is clear that existence will depend in a subtle way on the exponent $\theta(\zeta)$, particularly when $\theta$ contains several independently varying parameters (cf. Appendix II). Local existence in $x$ is probably easier to get, but not of much interest for applications to Painlevé equations. Thus, we restrict ourselves in this paper to the examination of various limiting cases and special examples. Other properties of Eqs. (3.33), (3.35) are under investigation.

\section{F. Special Solutions}

\section{(i) The linear limit}

Take $v=0$ and $a, b, c$, small. The usual procedure in solving (3.33) and (3.35) is to form the Neumann series. Here we keep only the terms linear in the parameters $a$, $b$, and $c$. We find

$$
\psi^{(1)} e^{\theta}=\left(\begin{array}{l}
1 \\
0
\end{array}\right)+\frac{a}{2 \pi i} \int_{C_{42}} \frac{\left(\begin{array}{l}
0 \\
1
\end{array}\right) e^{2 \theta}}{\xi-\zeta} d \xi+\frac{b}{2 \pi i} \int_{C_{46}} \frac{\left(\begin{array}{l}
0 \\
1
\end{array}\right) e^{2 \theta}}{\xi-\zeta} d \xi .
$$

Therefore from (3.36)

$$
\begin{aligned}
q & =\frac{a}{\pi} \int_{C_{42}} e^{8 i \xi^{3} / 3+2 i \xi x} d x+\frac{b}{\pi} \int_{C_{46}} e^{8 i \xi^{3 / 3}+2 i \xi x} d \xi \\
& =\left(a+\frac{b}{2}\right) \operatorname{Ai}(x)-\frac{i b}{2} \operatorname{Bi}(x)
\end{aligned}
$$


when $\operatorname{Ai}(x)$ and $\operatorname{Bi}(x)$ are the Airy functions. Note that when $q(x)$ is real, $c=-a^{*}$, $b=a^{*}-a$ to leading order and

$$
\begin{aligned}
q & =\frac{a+a^{*}}{2} \operatorname{Ai}(x)+\frac{i\left(a-a^{*}\right)}{2} \operatorname{Bi}(x) \\
& =\operatorname{Re} a \operatorname{Ai}(x)-\operatorname{Im} a \operatorname{Bi}(x) .
\end{aligned}
$$

(ii) Solutions which decay as $x \rightarrow+\infty$. Throughout this example, $v$ is taken to be zero. In this case, a one-parameter family of solutions of (3.1) has been studied by use of the Marchenko equation of the inverse scattering transform $([2,10-12])$, Eq. (2.14). Such techniques apply when $q(x) \rightarrow 0$ sufficiently rapidly as $x \rightarrow+\infty$; (3.1) then reduces to $q_{x x}=x q$ for large $x$. It can be proved that $q(x)$ has the expected asymptotic behavior,

$$
q(x) \sim \varrho \operatorname{Ai}(x)
$$

for some constant $\varrho$.

The rapid decay (3.39) ensures that the constructions of scattering theory can be used on the $x$-equation (3.3) at $x=+\infty$. In particular, the eigenfunction $v(x, \zeta)$ of (3.3) with asymptotic behavior

$$
v(x, \zeta) \sim e^{i \zeta x}\left(\begin{array}{l}
0 \\
1
\end{array}\right), \quad x \rightarrow+\infty
$$

is defined for large enough $x$, and it admits the triangular representation

$$
v(x, \zeta) e^{-i \zeta x}=\left(\begin{array}{l}
0 \\
1
\end{array}\right)+\int_{x}^{\infty} K(x, s) e^{i \zeta(s-x)} d s .
$$

By repeated integration by parts in (3.40), one may derive the asymptotic expansion

$$
v(x, \zeta) e^{-i \zeta x} \sim\left(\begin{array}{l}
0 \\
1
\end{array}\right)+\frac{C_{1}}{\zeta}+\ldots
$$

valid in the upper half $\zeta$-plane. Now, $v(x, \zeta) e^{-4 / 3 i \zeta^{3}}$ is precisely the solution $\psi^{(2)}$ of (3.2) on $\arg \zeta=0$, and since the expansion (3.41) is valid in $S_{1}, S_{2}, S_{3}$, the Stokes multiplier $b$ must vanish. In this case, (3.33) reduces to

$$
\begin{aligned}
\psi^{(1)}(\zeta) e^{\theta(\zeta)} & =\left(\begin{array}{l}
1 \\
0
\end{array}\right)+\frac{a}{2 \pi i} \int_{C_{42}} \frac{\psi^{(2)} e^{\theta}}{\xi-\zeta} d \xi \\
& =\left(\begin{array}{l}
1 \\
0
\end{array}\right)-a \psi^{(2)}(\zeta) e^{\theta(\zeta)}+\frac{a}{2 \pi i} \int_{-\infty}^{\infty} \frac{\psi^{(2)} e^{\theta}}{\xi-\zeta} d \xi
\end{aligned}
$$

Since $\psi^{(1)}+a \psi^{(2)}=\psi_{4}^{(1)}=M \psi^{(2)}(-\zeta)$, we may change $\zeta \rightarrow-\zeta$ in (3.42); then

$$
\left(\begin{array}{ll}
0 & 1 \\
1 & 0
\end{array}\right) \psi^{(2)}(\zeta) e^{-\theta(\zeta)}=\left(\begin{array}{l}
1 \\
0
\end{array}\right)+\frac{a}{2 \pi i} \int_{-\infty}^{\infty} \frac{\psi^{(2)} e^{\theta}}{\xi+\zeta} d \xi .
$$


Substitution of (3.40) into (3.43), followed by a Fourier transform [23], will recover the Marchenko equation (2.14). The constant $\varrho$ in the asymptotic form (3.39) of $q(x)$ coincides with the nonzero Stokes multiplier $a$.

In this example, therefore, one can see explicitly how the Stokes multipliers $a$ and $b$ characterize the Painlevé transcendent $q(x)$ :

$$
b=0 \text { implies } q(x) \rightarrow 0 \text { as } x \rightarrow+\infty,
$$

$a$ describes the asymptotic behavior, $q(x) \sim a \operatorname{Ai}(x)$.

It is shown in [11] that there is exactly one $q(x)$ satisfying these conditions. A more detailed analysis [11] of the Marchenko equation (2.14) reveals that $q(x)$ has no pole on the real axis when $-1<a<1$, whereas it has at least one real pole when $|a|>1$. A proof based on (3.43) would be quite analogous to the argument in [11]: for $|a|<1$, the inhomogeneous Eq. (3.43) can be solved for any real $x$ (remember that $x$ enters in $\theta$ ), while any $a$ with $|a|>1$ is eigenvalue of (3.43) for some real $x$. It may be possible, however, to go beyond the results of $[11,12]$. Since (3.43) is local in $x$, one can study the limits $x \rightarrow \pm \infty$ separately. This is relevant to the solution of the connection problem for the second Painleve transcendent $[10,11]$ : what is the behavior at $x=-\infty$ of the solution which goes as (3.39) at $+\infty$ ? The Marchenko equation (2.14), by the time $x$ goes to $-\infty$, contains information about $q(x)$ for all real $x$, and it is apparently difficult to extract the asymptotics at $-\infty$.

Connection formulas between singular points of Painlevé transcendents are important in several applications; in the Ising model, for example, the behavior near $x=0$ of the third transcendent is of physical interest $[4,13]$. We hope to return to these questions in a later paper.

(iii) The rational solutions: the "solitons" of the Painleve equations. In the inverse scattering transform, the solitons are associated with the bound states of the $x$ equations (3.3) which are located at the poles of the reflection coefficient in the upper half $\zeta$-plane. The analogue to the multisoliton solution is a class of rational solutions which are associated with the poles of the fundamental solution matrix $\Psi(\zeta, x)$ at $\zeta=0$. When $v=n$, we find these solutions by setting $a=b=c=0$. Then from (3.35),

$$
\psi^{(2)}(\zeta) e^{-\theta}=\left(\begin{array}{l}
0 \\
1
\end{array}\right)-\operatorname{Res}_{\zeta=0} \frac{\psi^{(2)} e^{-\theta}}{\xi-\zeta} .
$$

We mention that Eq. (3.44) will provide the rational solutions for the full class of equations of the Painlevé II family which is defined and discussed in Appendix II.

Near $\zeta=0$, the solution $\psi^{(2)}$ will be a linear combination of $\phi^{(1)}$ and $\phi^{(2)}$ as given in (3.13) and thus will have the form

$$
\psi^{(2)}=\zeta^{-n} \sum_{j=0}^{2 n-1} a_{j}\left(\begin{array}{c}
1 \\
(-1)^{j+1}
\end{array}\right) \zeta^{j}+\sum_{2 n}^{\infty} \mathbf{a}_{j} \zeta^{j} .
$$
Now since $a=b=c=0, \psi^{(2)} e^{-\theta}$ is meromorphic and tends to $\left(\begin{array}{l}0 \\ 1\end{array}\right)$ as $\zeta \rightarrow \infty$ and
therefore must have the form

$$
u=\psi^{(2)} e^{-\theta}=\zeta^{-n}\left(u_{0}+\zeta u_{1}+\ldots+\zeta^{n-1} u_{n-1}+\left(\begin{array}{l}
0 \\
1
\end{array}\right) \zeta^{n}\right)
$$


which follows from (3.44). From (3.6),

$$
q(x)=2 i u_{n-1,1}, \quad q^{2}=+2 i \frac{\partial}{\partial x} u_{n-1,2}
$$

where the subscripts 1 and 2 refer to the components of $u_{n-1}$. Set

$$
e^{\theta}=\sum_{j=0}^{\infty} T_{j} \zeta^{j}, \quad \theta=\sum_{j=0} i \omega_{2 j+1} \zeta^{2 j+1} / 2 j+1+i \zeta x .
$$

Expand $\psi^{(2)}$ in a series in $\zeta$,

$$
\psi^{(2)}=u e^{\theta}=\left(\frac{u_{0}}{\zeta^{n}}+\ldots+\frac{u_{n-1}}{\zeta}+\left(\begin{array}{l}
0 \\
1
\end{array}\right)\right) \sum_{0}^{\infty} T_{j} \zeta^{j} .
$$

We compute the coefficient of $\zeta^{k}$, and demand that it be orthogonal to $\left(\begin{array}{c}1 \\ (-1)^{n+k}\end{array}\right)$, $k=-n, \ldots, n-1$, as required by (3.45). With the notation $\xi_{j}=u_{1}^{j}+u_{2}^{j}, \eta_{j}=u_{1}^{j}-u_{2}^{j}$, the resulting $2 n$ equations can be written (if we use $T_{0}=1$ ):

$$
\begin{aligned}
& \xi_{0}=0 \\
& \eta_{0} T_{1}+\eta_{1}=0 \\
& \xi_{0} T_{2}+\xi_{1} T_{1}+\xi_{2}=0 \\
& \vdots \\
& \eta_{0} T_{2 n-1}+\ldots+\eta_{n-1} T_{n}=(-1)^{n} T_{n-1} .
\end{aligned}
$$

(3.48) decomposes into two separate systems for $\xi, \eta$ :

$$
\left(\begin{array}{l}
1 \\
T_{2} T_{1} 1 \\
T_{4} T_{3} T_{2} T_{1} 1 \\
T_{2 n-2} \ldots T_{n-1}
\end{array}\right)\left(\begin{array}{c}
\xi_{0} \\
\vdots \\
\vdots \\
\xi_{n-1}
\end{array}\right)=\left(\begin{array}{c}
0 \\
0 \\
-T_{1} \\
-T_{3} \\
-T_{n-2}
\end{array}\right) \text { or }\left(\begin{array}{c}
0 \\
0 \\
-1 \\
-T_{2} \\
-T_{n-2}
\end{array}\right)
$$

depending on whether $n$ is odd or even;

$$
\left.\left(\begin{array}{l}
T_{1} 10 \ldots \\
T_{3} T_{2} T_{1} 1 \ldots \\
T_{2 n-1} \ldots T_{n}
\end{array}\right)\left(\begin{array}{c}
\eta_{0} \\
\vdots \\
\eta_{n-1}
\end{array}\right)=\left(\begin{array}{l}
0 \\
1 \\
T_{2} \\
T_{n-1}
\end{array}\right)(n \text { odd }),\left(\begin{array}{l}
0 \\
T_{1} \\
T_{3} \\
T_{n-1}
\end{array}\right) \text { (n even }\right)
$$

It is useful to observe that the matrices in (3.49) are Wronskians. Indeed, $\frac{d}{d x} e^{\theta}=i \zeta e^{\theta}$, so that $T_{j x}=i T_{j-1}$. Hence, the derivative of each column is $i$ times the next column.

We now solve (3.49) for $\xi_{n-1}, \eta_{n-1}$ by Cramer's rule. The denominator determinants are denoted by $\Delta_{+}, \Delta_{-}$. By the Wronskian property, $\frac{d}{d x} \Delta_{ \pm}$is a single 
determinant in which the last column is replaced by its derivative, and it is easy to see that this derivative is in all cases proportional to the right-hand side of (3.49). Specifically,

$$
\xi_{n-1}=i \frac{\Delta_{+x}}{\Delta_{+}}, \quad \eta_{n-1}=-i \frac{\Delta_{-x}}{\Delta_{-}} .
$$

From (3.46) we find

$$
q=-\left(\ln \frac{\Delta_{+}}{\Delta_{-}}\right)_{x}, \quad q^{2}=-\left(\log \Delta_{+} \Delta_{-}\right)_{x x} .
$$

These are the solutions determined by Airault [3] through a series of recursion relations. The first two are, for the case $\theta=\frac{4}{3} i \zeta^{3}+i \zeta x$ :

$$
\begin{array}{ll}
n=1 . \quad \Delta_{+}=1, \quad \Delta_{-}=i x, \quad q=\frac{1}{x} . \\
n=2 . \quad \Delta_{+}=i x, \quad \Delta_{-}=-\frac{4}{3} i-\frac{i x^{3}}{3}, \quad q=-\frac{1}{x}+\frac{3 x^{2}}{4+x^{3}} .
\end{array}
$$

Observe that all these solutions satisfy a Riccati equation:

$$
q_{x}+q^{2}=-2\left(\log \Delta_{+}\right)_{x x} .
$$

An interesting class of solutions of the higher-order equations of the Painleve II family with $\theta$ given by (3.47) is obtained when $-2\left(\ln \Delta_{+}\right)_{x x}$ in (3.57) is

$$
\frac{n(n-1)}{x^{2}} \text {. }
$$

These solutions are related, by a Miura transformation, to certain rational solutions of the $\mathrm{KdV}$ hierarchy [25]. To get these solutions, let $\theta=i\left(\omega_{2 k+1} \zeta^{2 k+1}+\zeta x\right)$ and pick $n \leqq k+1$. In that case, all entries of $\Delta_{+}$arise from powers of $i \zeta x$ in the expansion (3.47) of $e^{\theta}$, and $\Delta_{+}$is the Wronskian determinant of $1, \frac{(i x)^{2}}{2 !}, \frac{(i x)^{4}}{4 !}, \ldots, \frac{(i x)^{2 n-2}}{(2 n-2) !}$. This is easily seen to be proportional to $x \cdot x^{2} \cdot x^{3} \ldots x^{n-1}$ $=x^{\frac{n(n-1)}{2}}$. Hence, $-2\left(\log \Delta_{+}\right)_{x x}=n(n-1) / x^{2}$.

For each $n$, only one solution of

$$
q_{x}+q^{2}=\frac{n(n-1)}{x^{2}}, \quad n \geqq 1
$$

will also solve a Painlevé equation, and in fact it will solve all the equations of the Painlevé II family (Appendix II) in which the first nonzero power of $\zeta$ (besides $i \zeta x$ ) is at least $\zeta^{2 n-1}$.

(iv) Solutions with $v=\frac{2 n+1}{2}, j=0$. It was noted in $3 \mathrm{C}$ that this is an exceptional case. We begin with a detailed description of the situation for $v=\frac{1}{2}$. 
According to (3.15) and (3.16), $j=0$ implies

$q_{x}+q^{2}+\frac{x}{2}=0$.

Let $q(x)=\frac{d}{d x} \ln \chi$; then

$$
\chi_{x x}=-\frac{x}{2} \chi,
$$

whose general solution is

$$
\chi=C_{1} \mathrm{Ai}\left(-2^{-1 / 3} x\right)+C_{2} \operatorname{Bi}\left(-2^{-1 / 3} x\right) .
$$

The solutions of (3.2), (3.3) can be verified to be

$$
F(\zeta, x)=\frac{\alpha_{0}}{2} \zeta^{1 / 2}\left(\begin{array}{c}
\left(1-\frac{i q}{\zeta}\right) \operatorname{Ai}(z)-2^{-1 / 3} \frac{i}{\zeta} \operatorname{Ai}^{\prime}(z) \\
\left(1+\frac{i q}{\zeta}\right) \operatorname{Ai}(z)+2^{-1 / 3} \frac{i}{\zeta} \operatorname{Ai}^{\prime}(z)
\end{array}\right)
$$

and $G(\zeta, x)$, which contains $\mathrm{Bi}$ instead of $\mathrm{Ai}$; here

$$
z=e^{i \pi}\left(2^{2 / 3} \zeta^{2}+2^{-1 / 3} x\right), \alpha_{0}=2^{1 / 6} \sqrt{\pi} e^{i \pi / 4} .
$$

Using the asymptotic properties of Airy functions, one may verify that

$$
\begin{aligned}
& \Psi_{1}=(-i F+G, F-i G), \\
& \Psi_{2}=(-2 i F, F-i G)=\Psi_{1}\left(\begin{array}{rr}
1 & 0 \\
-i & 1
\end{array}\right), \\
& \Psi_{3}=(-2 i F,-F-i G)=\Psi_{2}\left(\begin{array}{rr}
1 & -i \\
0 & 1
\end{array}\right), \\
& \Psi_{4}=(-i F-G,-F-i G)=\Psi_{3}\left(\begin{array}{rr}
1 & 0 \\
-i & 1
\end{array}\right), \\
& \Psi_{5}=(-i F-G,-2 F)=\Psi_{4}\left(\begin{array}{rr}
1 & -i \\
0 & 1
\end{array}\right), \\
& \Psi_{6}=(i F-G,-2 F)=\Psi_{5}\left(\begin{array}{rr}
1 & 0 \\
-i & 1
\end{array}\right), \\
& \Psi_{7}=(i F-G,-F+i G)=\Psi_{6}\left(\begin{array}{rr}
1 & -i \\
0 & 1
\end{array}\right),
\end{aligned}
$$

from which it is evident that $a=b=c=-i$. The solution matrix $\Phi(\zeta, x)$ $=\left(\phi^{(1)}(\zeta, x), \phi^{(2)}(\zeta, x)\right)$ defined by its behavior at the origin $\zeta=0$ is

$$
\begin{aligned}
\phi^{(1)} & =\frac{\left(K_{1} F+K_{2} G\right)}{\beta_{0}\left(K_{1} C_{2}-K_{2} C_{1}\right)}, \text { any } K_{2} / K_{1} \neq C_{2} / C_{1} \\
\phi^{(2)} & =\frac{2}{\alpha_{0}}\left(C_{1} F+C_{2} G\right),
\end{aligned}
$$


where $\beta_{0}=i \alpha_{0} / 2^{4 / 3} \pi$. The indeterminacy described in $3 \mathrm{C}$ is seen in the arbitrariness of $K_{1}, K_{2}$.

Finally, note that since $\operatorname{Ai}(z)$ and $\operatorname{Bi}(z)$ are single valued functions of $\zeta$, the only contribution to the jump matrix $J$ is from the square root $\zeta^{-1 / 2}$. Hence, $J=-I$. We next describe the general case $v=\frac{2 n+1}{2}(n \geqq 0)$.

Let $A(x, \zeta)$ be a fixed solution of $A_{x x}=\left(-\frac{1}{2} x-\zeta^{2}\right) A$. The solution $\phi^{(2)}$ of (3.2) is sought in the form

$$
\alpha_{0} \zeta^{1 / 2}\left\{A(x, \zeta)\left(C_{0}+\frac{C_{1}}{\zeta}+\ldots+\frac{C_{n+1}}{\zeta^{n+1}}\right)+A_{x}(x, \zeta)\left(\frac{d_{1}}{\zeta}+\ldots+\frac{d_{n+1}}{\zeta^{n+1}}\right)\right\}
$$

where $C_{0}=\left(\begin{array}{c}1 \\ (-1)^{n}\end{array}\right), d_{1}=i\left(\begin{array}{c}1 \\ (-1)^{n+1}\end{array}\right)$, and the coefficients follow the alternating $\left(\begin{array}{r}1 \\ \pm 1\end{array}\right)$ pattern. By definition, the leading power in $\phi^{(2)}$ is $\zeta^{\frac{2 n+1}{2}}$. One must therefore equate to zero the coefficients of $\zeta^{-\frac{2 n+1}{2}}, \ldots, \zeta^{\frac{2 n-1}{2}}$ in $(3.53)$; this gives $2 n+1$ equations for $C_{1}, \ldots, C_{n+1}, d_{2}, \ldots, d_{n+1}$, and the coefficients of these equations will involve derivatives $\left.\frac{\partial^{j}}{\partial \zeta^{i}} A(x, \zeta)\right|_{\zeta=0}$. The coefficient of $\zeta^{\frac{2 n+1}{2}}$ in (3.53) is $e^{u(x)}$ [see (3.13)], and its log derivative is the required $q(x)$.

A concise expression is afforded by

$$
q(x)=-\frac{d}{d x} \ln \frac{W\left(\gamma, \ldots, \gamma^{(n-1)}\right)}{W\left(\gamma, \ldots, \gamma^{(n)}\right)},
$$

where $\gamma(x)=A(x, 0)$, and $W$ is the Wronskian determinant. (These solutions were discovered by Airault [3].)

\section{Painlevé III; Solution of an Initial Value Problem}

\section{A. Outline}

In Appendix I, we show that the equation

$$
\left(x u_{x}\right)_{x}=-4 \sinh u
$$

a special case of the Painleve equation of the third kind, is the integrability condition for

$$
\begin{aligned}
& v_{1 \zeta}=\left(-i x+\frac{i}{\zeta^{2}} \cosh u\right) v_{1}+\left(\frac{-x u_{x}}{2 \zeta}+\frac{i}{\zeta^{2}} \sinh u\right) v_{2}, \\
& v_{2 \zeta}=\left(\frac{-x u_{x}}{2 \zeta}-\frac{i}{\zeta^{2}} \sinh u\right) v_{1}+\left(i x-\frac{i}{\zeta^{2}} \cosh u\right) v_{2},
\end{aligned}
$$

and

$$
\begin{aligned}
& v_{1 x}=-i \zeta v_{1}+q v_{2}, \quad q=-u_{x} / 2, \\
& v_{2 x}=q v_{1}+i \zeta v_{2} .
\end{aligned}
$$


In Appendix III, we show how these equations relate to the work of Satō et al. [7]. Following the steps described in Sect. 3, we give the general solution of (4.1). The principal difference between (3.2) and (4.2) is that the former has one regular and one irregular singular point, while the latter has two irregular singular points, one at $\zeta=\infty$ and the other at $\zeta=0$.

\section{B. Step 1: The Direct Transform and Properties of the Transform Data}

It is straightforward to write down the asymptotic forms $\tilde{\psi}^{(1)}, \tilde{\psi}^{(2)}$ of two linearly independent solutions of (4.2) as $\zeta \rightarrow \infty$ :

$$
\begin{aligned}
& \tilde{\psi}^{(1)}(\zeta, x)=e^{-i \zeta x-i / \zeta}\left\{\left(\begin{array}{l}
1 \\
0
\end{array}\right)+\frac{1}{\zeta}\left(\begin{array}{c}
-i x q^{2} / 2+i(1-\cosh u) \\
i q / 2
\end{array}\right)+\ldots\right\}, \\
& \tilde{\psi}^{(2)}(\zeta, x)=e^{i \zeta x+i / \zeta}\left\{\left(\begin{array}{l}
0 \\
1
\end{array}\right)+\frac{1}{\zeta}\left(\begin{array}{c}
-i q / 2 \\
i x q^{2} / 2-i(1-\cosh u)
\end{array}\right)+\ldots\right\}
\end{aligned}
$$

Note that, from (4.1), $x q^{2} / 2+\cosh u-1=\frac{1}{2} \int^{x} q^{2}+$ const. We have found it convenient for reasons of symmetry to include the $i / \zeta$ term in the exponent of (4.4). At $\zeta=0$, two linearly independent solutions $\phi^{(1)}, \phi^{(2)}$ have the asymptotic behavior:

$$
\begin{aligned}
& \tilde{\phi}^{(1)}(\zeta, x)=e^{-i \zeta x-i / \zeta}\left(\begin{array}{c}
\cosh \frac{u}{2} \\
-\sinh \frac{u}{2}
\end{array}\right) \quad(1+\ldots), \\
& \tilde{\phi}^{(2)}(\zeta, x)=e^{i \zeta x+i / \zeta}\left(\begin{array}{l}
-\sinh \frac{u}{2} \\
\cosh \frac{u}{2}
\end{array}\right) \quad(1+\ldots) .
\end{aligned}
$$

In particular, we note that, if we write $\tilde{\Psi}=\left(\tilde{\psi}^{(1)}, \tilde{\psi}^{(2)}\right)$ and $\tilde{\Phi}=\left(\tilde{\phi}^{(1)}, \tilde{\phi}^{(2)}\right)$, the following relations hold:

$$
M \tilde{\Psi}(-\zeta, x)=\tilde{\Psi}(\zeta, x) M, \quad M \tilde{\Phi}(-\zeta, x)=\tilde{\Phi}(\zeta, x) M
$$

and

$$
N \tilde{\Psi}\left(-\frac{1}{\zeta x}, x\right)=\tilde{\Phi}(\zeta, x)\left(\begin{array}{rr}
0 & 1 \\
-1 & 0
\end{array}\right),
$$

where

$$
M=\left(\begin{array}{ll}
0 & 1 \\
1 & 0
\end{array}\right) \text { and } N=\left(\begin{array}{cc}
\cosh \frac{u}{2} & -\sinh \frac{u}{2} \\
-\sinh \frac{u}{2} & \cosh \frac{u}{2}
\end{array}\right)\left(\begin{array}{rr}
0 & 1 \\
-1 & 0
\end{array}\right) .
$$


Indeed, from Eqs. (4.2), (4.3) one can show that if $\Psi(\zeta, x)=\left(\psi^{(1)}(\zeta, x), \psi^{(2)}(\zeta, x)\right)$ is a solution, then so is

$$
M \Psi(-\zeta, x) \text { and } N \Psi\left(-\frac{1}{\zeta x}, x\right) .
$$

From the asymptotic expressions (4.4), (4.5), we observe that the lines on which the asymptotic solutions change from recessive to dominant and vice versa, the anti-Stokes lines, are $\arg \zeta=n \pi, n=-2,-1,0,1,2, \ldots$. Accordingly, we designate the sectors $-2 \pi \leqq \arg \zeta<-\pi,-\pi \leqq \arg \zeta<0,0 \leqq \arg \zeta<\pi, \pi \leqq \arg \zeta<2 \pi$, and $2 \pi \leqq \arg \zeta<3 \pi$ as $S_{-2}, S_{-1}, S_{1}, S_{2}, S_{3}$, respectively, and define $\Psi_{j}(\zeta, x)$ and $\Phi_{j}(\zeta, x)$, $j=-2,-1,1,2,3$ to be the solutions in these sectors which have the asymptotic behaviors (4.4) and (4.5) on the initial ray of each sector. For convenience, we omit the subscript 1 when referring to the first sector.

We will now show that all the Stokes multiplier matrices for the $\Psi_{j}$ are $\left(\begin{array}{ll}1 & a \\ 0 & 1\end{array}\right)$ or $\left(\begin{array}{ll}1 & 0 \\ a & 1\end{array}\right)$ and for the $\Phi_{j},\left(\begin{array}{ll}1 & \bar{a} \\ 0 & 1\end{array}\right)$ or $\left(\begin{array}{ll}1 & 0 \\ \bar{a} & 1\end{array}\right)$. First, if $x<0, \tilde{\psi}^{(1)}$ and $\tilde{\phi}^{(1)}$ are recessive in $S_{-2}, S_{1}, S_{3}$, and dominant in $S_{-1}, S_{2}, \tilde{\psi}^{(2)}, \tilde{\phi}^{(2)}$ are recessive in $S_{-1}, S_{2}$ and dominant in $S_{-2}, S_{1}, S_{3}$. Therefore,

$$
\begin{aligned}
& \Psi_{-1}=\Psi_{-2}\left(\begin{array}{cc}
1 & a_{-2} \\
0 & 1
\end{array}\right), \quad \Psi=\Psi_{-1}\left(\begin{array}{cc}
1 & 0 \\
a_{-1} & 1
\end{array}\right) \\
& \Psi_{2}=\psi\left(\begin{array}{ll}
1 & a \\
0 & 1
\end{array}\right), \quad \psi_{3}=\psi_{2}\left(\begin{array}{cc}
1 & 0 \\
a_{2} & 1
\end{array}\right) .
\end{aligned}
$$

From the symmetry (4.9), we have that

$$
\begin{aligned}
& M \Psi\left(\zeta e^{-i \pi}, x\right) M^{-1}=\Psi_{2}(\zeta, x), \\
& M \Psi_{2}\left(\zeta e^{-i \pi}, x\right) M^{-1}=\Psi_{3}(\zeta, x) .
\end{aligned}
$$

To see this, observe that the left-hand sides of (4.11) are solutions of (4.2) with the required asymptotic properties on the initial rays of the respective sectors $S_{2}$ and $S_{3}$. Substitute in (4.11b) from (4.10) and find

$$
M\left(\begin{array}{ll}
1 & a \\
0 & 1
\end{array}\right)=\left(\begin{array}{ll}
1 & 0 \\
a_{2} & 1
\end{array}\right) M
$$

whence $a_{2}=a$. By a similar argument, $a_{-2}=a_{-1}=a$. We can also prove that $\bar{a}$ is the only Stokes multiplier connecting the matrices $\Phi_{j}$. If $x>0$, the Stokes multiplier matrices are the transposes of those for the case $x<0$ :

$$
\Psi_{2}=\Psi\left(\begin{array}{ll}
1 & 0 \\
a & 1
\end{array}\right), \quad \Psi_{3}=\Psi_{2}\left(\begin{array}{ll}
1 & a \\
0 & 1
\end{array}\right) .
$$

The matrices $\Psi$ and $\Phi$ will, in general, not be single-valued. We next calculate the monodromy matrices $J_{\mp}$ and $\bar{J}$ defined by the relations

$$
\Psi\left(\zeta e^{2 \pi i}\right)=\Psi(\zeta) J_{\mp}, \quad \Phi\left(\zeta e^{2 \pi i}\right)=\Phi(\zeta) \bar{J}
$$


in terms of the Stokes multipliers $a$ and $\bar{a}$. The subscripts -, + refer to the cases $x<0$ and $x>0$, respectively. From (4.10) (the case $x<0$ ), we have

$$
\Psi_{3}\left(\zeta e^{2 \pi i}\right)\left(\begin{array}{cc}
1 & -a \\
-a & 1+a^{2}
\end{array}\right)=\Psi(\zeta) J_{-} .
$$

Now let $\zeta \in S_{1}$ tend to infinity; then

$$
J_{-}=\left(\begin{array}{cc}
1 & -a \\
-a & 1+a^{2}
\end{array}\right)
$$

since both $\Psi_{3}\left(\zeta e^{2 \pi i}\right)$ and $\Psi(\zeta)$ tend to $\tilde{\Psi}(\zeta)$. Similarly, if $x>0$,

$$
J_{+}=\left(\begin{array}{rr}
1+a^{2} & -a \\
-a & 1
\end{array}\right) \text {. }
$$

Also,

$$
\bar{J}=\left(\begin{array}{cc}
1 & -\bar{a} \\
-\bar{a} & 1+\bar{a}^{2}
\end{array}\right) .
$$

Finally, we specify the connection matrix $A$ between $\Psi$ and $\Phi$,

$$
\Psi=\Phi A, \quad A=\left(\begin{array}{ll}
\alpha & \beta \\
\gamma & \delta
\end{array}\right) .
$$

From the normalizations of $\Psi$ and $\Phi, \alpha \delta-\beta \gamma=1$. Let us now derive relations between $\bar{J}, J_{-}, A$, for the case $x<0$. Let $\zeta \rightarrow \zeta e^{2 \pi i}$ in (4.18), and use (4.13) to find

$$
A J_{-}=\bar{J} A \text {. }
$$

Since $J_{-}$and $\bar{J}$ are similar, their traces are equal; from (4.15) and (4.17), $\bar{a}=s a$, $s= \pm 1$. Comparison of the other entries in (4.19) gives

$$
\beta=s \gamma, \quad \alpha=s(\delta+a \gamma) .
$$

Among the transform data, therefore, there are only two independent constants which we take to be $a$ and $\frac{\gamma}{\delta}$. The reason for the sign parameter $s$ is discussed later.

Similarly, if $x>0$, we find

$$
A J_{+}=\bar{J} A \text {, }
$$

from which we have

$$
\bar{a}=s a, \quad \alpha=s \delta, \quad \beta=s(\gamma+a \delta) .
$$

\section{C. Step 2: The x-Dependence of the Transform Data}

Arguments which exactly parallel those given in Sect. 3 show that:

(1) If (4.2) and (4.3) and therefore (4.1) hold, the transform data, which consist of the Stokes multiplier matrix $\left(\begin{array}{ll}1 & a \\ 0 & 1\end{array}\right)$, the monodromy matrices $J_{+}, J_{-}, \bar{J}$ and the connection matrix $A$, are independent of $x$. 
(2) Conversely, if the transform data are independent of $x$, and if we specify functions $\Psi_{j}, \Phi_{j}$ to be (a) connected as in (4.10), (4.13), and (4.18), with (b) the symmetry properties stemming from (4.9), and (c) with the asymptotic expansions (4.4) and (4.5), then the functions $\Psi_{j}, \Phi_{j}$ satisfy differential equations in $\zeta$ and $x$ which have precisely the form (4.2), (4.3). The coefficients in the equations are directly related to the coefficients in the specified asymptotic expansions.

\section{D. Step 3: The Inverse Transform}

Here we show how to derive, at any fixed position $x$, a singular integral equation for the matrix function $\Psi(\zeta, x)$ from which one can, in principle, construct the function $\Psi(\zeta, x)$ and the Eq. (4.2) which it satisfies. Since the coefficients in this equation are functions of the solution $u(x)$ of (4.1), we have therefore found $u(x)(\bmod 2 \pi i)$ for any given $x$. We first look at the case $x<0$. Consider

$$
\int_{C_{1}} \frac{\psi^{(1)} e^{\theta}}{\xi-\zeta} d \xi \quad \text { with } \quad \theta=i \xi x+\frac{i}{\xi}
$$

The reader should refer to Fig. 4 for the definition of the contours. The contour $\Gamma$ has a large radius, the contour $\gamma$ a small one. Let $\zeta \in S_{1}$, outside the circle designated by $\lambda$.

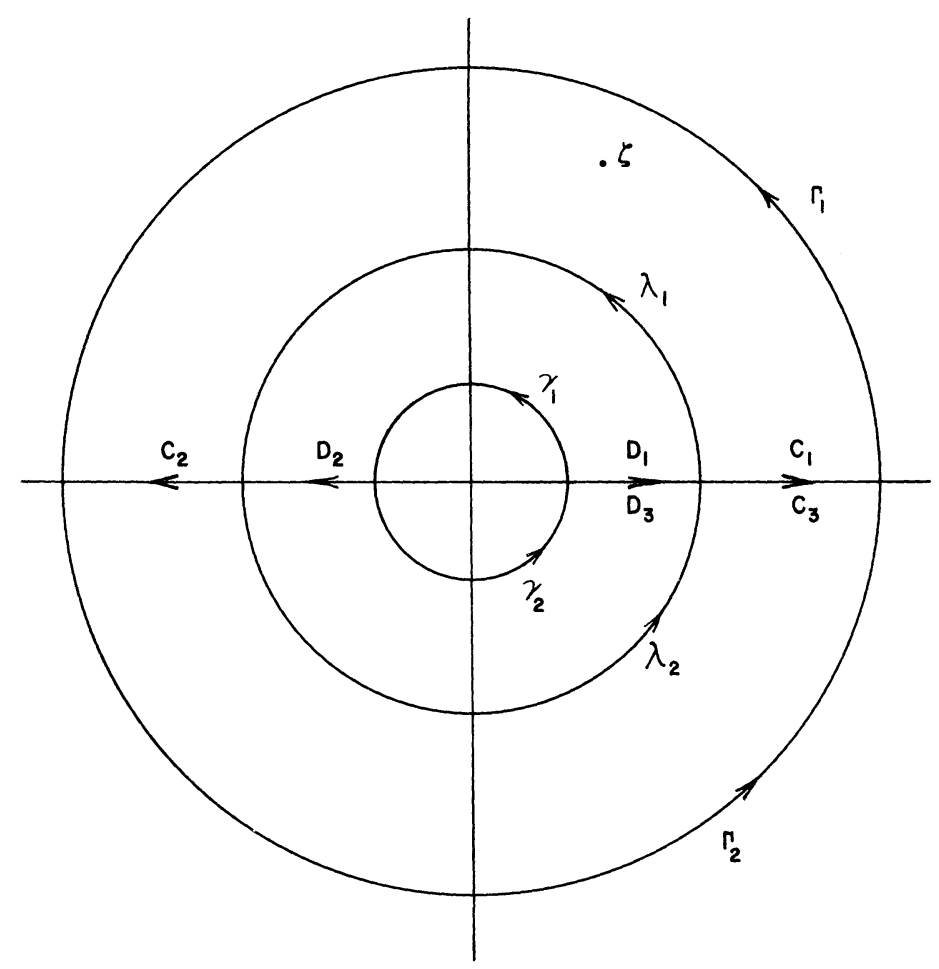

Fig. 4. The contours in the $\zeta$-plane for the inverse problem for Painleve III, $x<0$ 
Using Cauchy's theorem, we obtain

$$
\int_{C_{1}} \frac{\psi^{(1)} e^{\theta}}{\xi-\zeta} d \xi+\pi i\left(\begin{array}{l}
1 \\
0
\end{array}\right)=2 \pi i \psi^{(1)} e^{\theta}+\int_{C_{2}} \frac{\psi^{(1)} e^{\theta}}{\xi-\zeta} d \xi+\int_{\lambda_{1}} \frac{\psi^{(1)} e^{\theta}}{\xi-\zeta} d \xi .
$$

But $\psi^{(1)}=\psi_{2}^{(1)}$ and

$$
\int_{C_{2}} \frac{\psi_{2}^{(1)} e^{\theta}}{\xi-\zeta} d \xi+\pi i\left(\begin{array}{l}
1 \\
0
\end{array}\right)=\int_{C_{3}} \frac{\psi_{2}^{(1)} e^{\theta}}{\xi-\zeta} d \xi+\int_{\lambda_{2}} \frac{\psi_{2}^{(1)} e^{\theta}}{\xi-\zeta} d \xi
$$

We add the two equations to obtain

$$
\psi^{(1)} e^{\theta}=\left(\begin{array}{l}
1 \\
0
\end{array}\right)+\frac{a}{2 \pi i} \int_{C_{1}} \frac{\psi^{(2)} e^{\theta}}{\xi-\zeta} d \xi-\frac{1}{2 \pi i} \int_{\lambda} \frac{\psi^{(1)} e^{\theta}}{\xi-\zeta} d \xi,
$$

upon making use of the relations $\psi_{2}^{(1)}=\psi^{(1)}$ and [from $\left.(4.13)\right] \psi^{(1)}\left(\zeta e^{2 \pi i}\right)=\psi^{(1)}(\zeta)$ $-a \psi^{(2)}(\zeta) . \lambda$ is the union of the two contours $\lambda_{1}$ and $\lambda_{2}$. We will see later that, in the linear limit, the second term on the RHS of (4.26) gives rise to the solution $K_{0}(4 \sqrt{-x})$ of the linearized (4.1), and the third term, which will be associated with the off-diagonal terms in the connection matrix $A$, gives rise to the solution $I_{0}(4 \sqrt{-x})$ of the linearized (4.1).

Using (4.18), we find

$$
\int_{\lambda} \frac{\psi^{(1)} e^{\theta}}{\xi-\zeta} d \xi=\frac{1}{\delta} \int_{\lambda_{1}} \frac{\phi^{(1)} e^{\theta}}{\xi-\zeta} d \xi+\frac{\gamma}{\delta} \int_{\lambda_{1}} \frac{\psi^{(2)} e^{\theta}}{\xi-\zeta} d \xi+\int_{\lambda_{2}} \frac{\psi^{(1)} e^{\theta}}{\xi-\zeta} d \xi,
$$

which, because $\phi^{(1)} e^{\theta}$ is bounded on the contours $\gamma_{1}$ and $\gamma_{2}$ whose radius is arbitrarily small, is equal to

$$
-\frac{1}{\delta} \int_{D_{1}} \frac{\phi^{(1)} e^{\theta}}{\xi-\zeta} d \xi+\frac{1}{\delta} \int_{D_{2}} \frac{\phi^{(1)} e^{\theta}}{\xi-\zeta} d \xi+\frac{\gamma}{\delta} \int_{\lambda_{1}} \frac{\psi^{(2)} e^{\theta}}{\xi-\zeta}+\int_{\lambda_{2}} \frac{\psi^{(1)} e^{\theta}}{\xi-\zeta} d \xi .
$$

But $\phi_{2}^{(1)}=\phi^{(1)}$, and continuing the second integral into the lower half plane and using the relations $\phi^{(1)}\left(\zeta e^{2 \pi i}\right)=\phi^{(1)}(\zeta)-s a \phi^{(2)}(\zeta)$ and $\psi^{(1)}-\frac{1}{\delta} \phi^{(1)}=\frac{\gamma}{\delta} \psi^{(2)}$, we find

$$
\int_{\lambda} \frac{\psi^{(1)} e^{\theta}}{\xi-\zeta} d \xi=-\frac{s a}{\delta} \int_{D_{1}} \frac{\phi^{(2)} e^{\theta}}{\xi-\zeta} d \xi+\frac{\gamma}{\delta} \int_{\lambda} \frac{\psi^{(2)} e^{\theta}}{\xi-\zeta} d \xi
$$

Finally using $\phi^{(2)}=-\beta \psi^{(1)}+\alpha \psi^{(2)}$ and replacing $\alpha$ and $\beta$ from (4.20), we find

$$
\begin{aligned}
\psi^{(1)}(\zeta) e^{\theta}= & \left(\begin{array}{l}
1 \\
0
\end{array}\right)+\frac{a}{2 \pi i} \int_{0}^{\infty} \frac{\psi^{(2)}(\xi) e^{\theta}}{\xi-\zeta}-\frac{\gamma}{2 \pi i \delta} \int_{\lambda} \frac{\psi^{(2)}(\xi) e^{\theta}}{\xi-\zeta} d \xi \\
& +\frac{a^{2} \gamma}{\delta} \int_{D_{1}} \frac{\psi^{(2)}(\xi) e^{\theta}}{\xi-\zeta} d \xi-\frac{a \gamma}{2 \pi i \delta} \int_{D_{1}} \frac{\psi^{(1)}(\xi) e^{\theta}}{\xi-\zeta} d \xi
\end{aligned}
$$

which together with $M \psi^{(2)}\left(\zeta e^{i \pi}\right)=\psi^{(1)}(\zeta)$ defines the solution matrix $\Psi(\zeta, x)$.

(4.27) is a singular integral equation in which the parameter $x<0$ only appears in the exponent $\theta(\xi)=i \xi x+\frac{i}{\xi}$. The initial conditions $u\left(x_{0}\right), u_{x}\left(x_{0}\right)$ are represented by the parameters $a$ and $\frac{\gamma}{\delta}$. Notice that the sign parameter $s$ has disappeared 
altogether and plays no role in the determination of the solution $u(x)$ of (4.1). Its appearance in the transform data is a consequence of the symmetry

$$
u \rightarrow-u, \quad v \rightarrow\left(\begin{array}{rr}
1 & 0 \\
0 & -1
\end{array}\right) v \quad \text { or } \quad\left(\begin{array}{rr}
-1 & 0 \\
0 & 1
\end{array}\right) v
$$

present in (4.2), (4.3). This is reflected in (4.27): note that if $\psi^{(1)}\left(\zeta, a, \frac{\gamma}{\delta}\right), \psi^{(2)}\left(\zeta, a, \frac{\gamma}{\delta}\right)$ is a solution, then so is $\left(\begin{array}{rr}1 & 0 \\ 0 & -1\end{array}\right) \psi^{(1)}\left(\zeta,-a,-\frac{\gamma}{\delta}\right)$ and $\left(\begin{array}{rr}-1 & 0 \\ 0 & 1\end{array}\right) \psi^{(2)}\left(\zeta,-a,-\frac{\gamma}{\delta}\right)$. Observe that this transformation also satisfies the symmetry condition, since $M\left(\begin{array}{rr}1 & 0 \\ 0 & -1\end{array}\right)=\left(\begin{array}{rr}-1 & 0 \\ 0 & 1\end{array}\right) M$.

We now apply the linear limit to (4.27) by taking $a$ and $\frac{\gamma}{\delta}$ to be small, neglecting all quadratic and cubic terms in the parameters and approximating $\psi^{(2)}$ by $\left(\begin{array}{l}0 \\ 1\end{array}\right) e^{\theta}$ to leading order. Then, using

$$
q(x)=\frac{-u_{x}}{2}=-\lim _{\zeta \rightarrow \infty} 2 i \zeta \psi_{, 2}^{(1)} e^{-\theta}=\lim _{\zeta \rightarrow \infty} 2 i \zeta \psi_{, 1}^{(2)} e^{\theta}
$$

(the subscripts refer to the components), we find that

$$
q(x)=\frac{a}{\pi} \int_{0}^{\infty} e^{2 i \xi x+2 i / \xi} d \xi-\frac{\gamma}{\pi \delta} \int_{\lambda} e^{2 i \xi x+2 i / \xi} d \xi
$$

Integrating with respect to $x$, setting $\xi=\frac{1}{\sqrt{-x}} e^{\phi}$ in the first integral, taking $\lambda$ to be the circle $|\xi|=\frac{1}{\sqrt{-x}}$ in the second and setting $\xi=\frac{1}{\sqrt{-x}} e^{i \phi}$, we find

$$
\begin{aligned}
u(x) & =\frac{i a}{\pi} \int_{0}^{\infty} \cos (4 \sqrt{-x} \sinh \phi) d \phi+\frac{\gamma}{\pi \delta} \int_{0}^{2 \pi} e^{4 \sqrt{-x} \sin \phi} d \phi \\
& =\frac{i a}{\pi} K_{0}(4 \sqrt{-x})+\frac{2 \gamma}{\delta} I_{0}(4 \sqrt{-x})
\end{aligned}
$$

We observe that if $u$ is real, $a$ is pure imaginary and $\frac{\gamma}{\delta}$ is real. This may be proved as follows. If $u$ is real, $M \psi^{*}\left(\zeta^{*}, x\right)$ is a solution if $\psi(\zeta, x)$ is. From the asymptotic behaviors we have that $M \Psi_{2}^{*}\left(\zeta^{*}\right)=\Psi_{-1}(\zeta) M$ and $M \Psi^{*}\left(\zeta^{*}\right)=\Psi(\zeta) M$. But $\Psi_{-1}=\Psi\left(\begin{array}{rr}1 & 0 \\ -a & 1\end{array}\right)$ and $\Psi_{2}=\Psi\left(\begin{array}{ll}1 & a \\ 0 & 1\end{array}\right)$ and from these relations we find $a^{*}=-a$. The reality of $\frac{\gamma}{\delta}$ follows from similar arguments.

The solution of (4.1) studied by Satō et al. corresponds to the case $\frac{\gamma}{\delta}=0$; that is, the inside-outside connection matrix $A$ is the identity $(s>0)$. From the remarks in example 2 of Sect. $3 \mathrm{~F}$, the reader may convince himself that this is also the case 
which can be treated by inverse scattering theory. In other words, the function $\psi^{(1)} e^{i / \zeta}$ is analytic in $\operatorname{Im} \zeta>0$ and in particular bounded as $\zeta \rightarrow 0$ only if $\psi^{(1)}$ contains no component of $\phi^{(2)}$, which from (4.18) and (4.20) means that $\beta=\gamma=0$.

When $x>0$, the derivation of the singular integral equation is simpler and can be accomplished without the contour $\lambda$. Let $\zeta \in S_{2}$.

Use Cauchy's theorem to express $\int_{C_{1}} \frac{\psi^{(1)} e^{\theta}}{\xi-\zeta} d \xi$ in terms of an integral along $C_{2}$. Because this will involve an integral along $\gamma_{1}$ on which contour $\phi^{(2)} e^{\theta}$ is

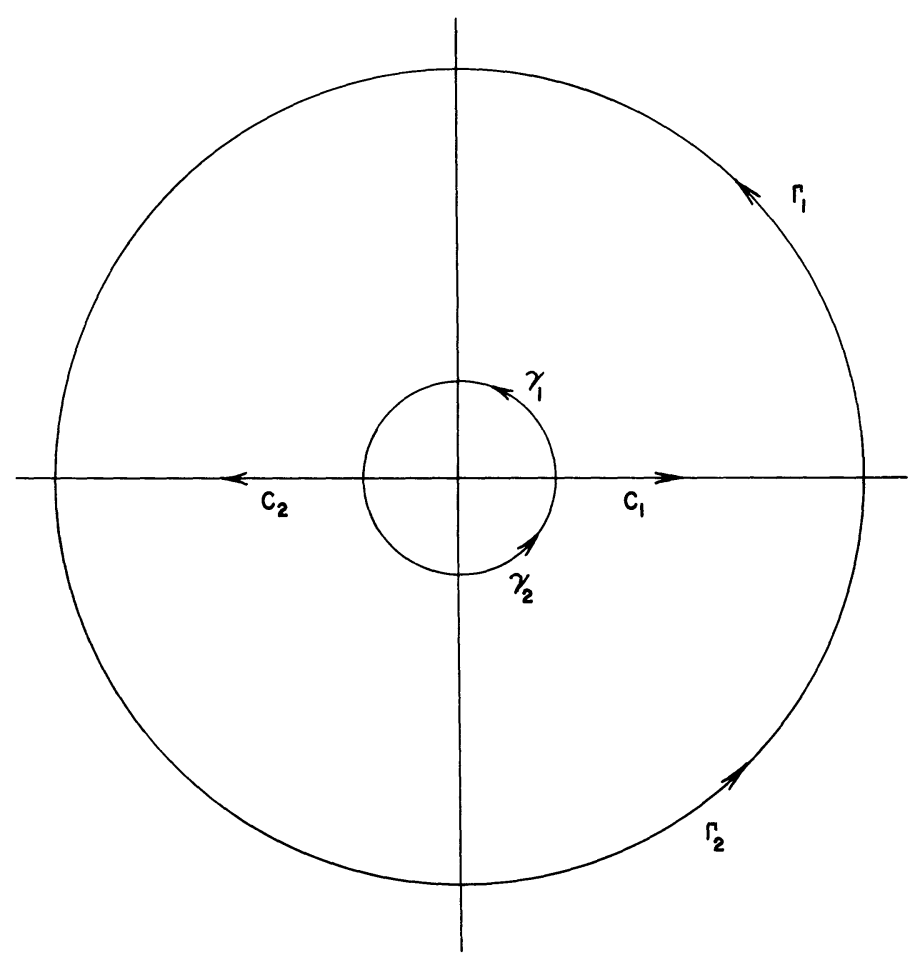

Fig. 5. The contours in the $\zeta$-plane for the inverse problem for Painleve III, $x>0$

exponentially large, we must subtract an appropriate amount of $\psi^{(2)}$ from $\psi^{(1)}$ in order that the $\phi^{(2)}$ component be eliminated:

$$
\begin{aligned}
\int_{C_{1}} \frac{\psi^{(1)} e^{\theta}}{\xi-\zeta} d \xi & =\int_{C_{1}} \frac{\psi^{(1)}-\frac{\gamma}{\delta} \psi^{(2)}}{\zeta-\zeta} e^{\theta} d \xi+\frac{\gamma}{\delta} \int_{C_{1}} \frac{\psi^{(2)} e^{\theta}}{\xi-\zeta} d \xi \\
& =\int_{C_{2}} \frac{\psi^{(1)}-\frac{\gamma}{\delta} \psi^{(2)}}{\xi-\zeta} e^{\theta} d \xi-\pi i\left(\begin{array}{l}
1 \\
0
\end{array}\right)+\frac{\gamma}{\delta} \int_{C_{1}} \frac{\psi^{(2)} e^{\theta}}{\xi-\zeta} d \xi
\end{aligned}
$$


But $\psi^{(2)}=\psi_{2}^{(2)}, \psi^{(1)}=\psi_{2}^{(1)}-a \psi_{2}^{(2)}$. Thus we have

$$
\int_{C_{1}} \frac{\psi^{(1)} e^{\theta}}{\xi-\zeta} d \xi=\int_{C_{2}} \frac{\psi_{2}^{(1)}-\left(a+\frac{\gamma}{\delta}\right) \psi_{2}^{(2)}}{\zeta-\zeta} e^{\theta} d \xi-\pi i\left(\begin{array}{l}
1 \\
0
\end{array}\right)+\frac{\gamma}{\delta} \int_{C_{1}} \frac{\psi_{2}^{(2)} e^{\theta}}{\xi-\zeta} d \xi .
$$

Now $\psi_{2}^{(1)} e^{\theta}, \phi^{(1)} e^{\theta}, \phi^{(2)} e^{\theta}$ are bounded on $\Gamma_{2}$ and $\gamma_{2}$ respectively, and thus $\int_{C_{2}} \frac{\psi_{2}^{(1)} e^{\theta}}{\xi-\zeta}$ can be continued to $\arg \zeta=2 \pi$. On this line, $\psi_{2}^{(1)}\left(\zeta e^{2 \pi i}\right)=\psi^{(1)}\left(\zeta e^{2 \pi i}\right)+a \psi^{(2)}\left(\zeta e^{2 \pi i}\right)$ $=\left(1+a^{2}\right) \psi^{(1)}(\zeta)-a \psi^{(2)}(\zeta)+a \psi^{(2)}(\zeta)-a^{2} \psi^{(1)}(\zeta)=\psi^{(1)}(\zeta)$. Thus the integrals $\int_{C_{1}} \frac{\psi^{(1)} e^{\theta}}{\xi-\zeta} d \xi$ on $\arg \xi=0$ and $\int_{C_{1}} \frac{\psi_{2}^{(1)} e^{\theta}}{\xi-\zeta} d \xi$ on $\arg \xi=2 \pi$ cancel. We find

$$
\psi_{2}^{(1)}(\zeta) e^{\theta}=\left(\begin{array}{l}
1 \\
0
\end{array}\right)+\frac{a}{2 \pi i} \int_{0}^{-\infty} \frac{\psi_{2}^{(2)}(\zeta) e^{\theta}}{\xi-\zeta} d \xi-\frac{\gamma}{2 \pi i \delta} \int_{-\infty}^{\infty} \frac{\psi_{2}^{(2)}(\zeta) e^{\theta}}{\zeta-\zeta} d \xi
$$

Taking the linear limit, we obtain (as expected)

$$
u(x)=a H_{0}^{(2)}(4 \sqrt{x})+\frac{\gamma}{\delta} J_{0}(4 \sqrt{x}) \quad \text { with } \quad x>0
$$

the solution of the linearized (4.1).

We remark, in conclusion, that just as the contours involved in the singular integral equations for finding the solution of Painleve II are those used in the representations of Airy functions, so the contours appropriate for (4.1) are familiar from the representations of Bessel functions.

\section{Hamiltonian Systems}

Another property shared by monodromy - and spectrum - preserving deformations is that the deformation equations can be written as completely integrable Hamiltonian systems. The modified Korteweg-deVries equation (2.9) can be written $q_{t}=\partial / \partial x \frac{\delta H}{\delta q}$, with $H=\frac{1}{2} \int_{-\infty}^{\infty}\left(q_{x}^{2}+q^{4}\right) d x$. It can be shown [24] that the mapping to scattering data is canonical and that suitable combinations of the scattering data are action - angle variables. In contrast, Eqs. (1.2) and (1.4) can each be written as a four-dimensional Hamiltonian system with $x$ playing the role of time.

Consider (1.2) and let

$$
p_{1}=q_{x}, q_{1}=q, p_{2}=\frac{1}{2} \int_{x_{0}}^{x} q^{2} d x, q_{2}=x .
$$

Then

$$
H\left(p_{1}, q_{1}, p_{2}, q_{2}\right)=\frac{1}{2} p_{1}^{2}-\frac{1}{2} q_{2} q_{1}^{2}+p_{2}-\frac{1}{2} q_{1}^{4}+v q_{1}
$$

is the Hamiltonian. It can be verified directly that $H$ is constant and that Hamilton's equations are satisfied by (1.2). But we have already shown that each 
piece of monodromy data $(a, b, c, j, \alpha, \beta, \gamma, \delta)$ is also a constant of the motion and thus

$$
a_{x}=\{H, a\}=0,
$$

where $\{H, a\}$ is the usual Poisson bracket. Therefore we have two independent constants of the motion in involution and thus the system is exactly integrable.

Similarly, for (1.4), set

$$
p_{1}=x u_{x}, q_{1}=u, p_{2}=\frac{1}{2} \int_{x_{0}}^{x} u_{x}^{2} d x, q_{2}=x
$$

and

$$
H\left(p_{1}, q_{1}, p_{2}, q_{2}\right)=\frac{1}{2 q_{2}} p_{1}^{2}+p_{2}+4 \cosh q_{1} .
$$

Again any one of the monodromy data is an independent constant of the motion which commutes with $H$ and the system is again integrable.

The Hamiltonian property carries through for any one of the members of the Painlevé II family discussed in Appendix II.

We have not as yet examined in what sense the transformation from $x, q, q_{x}$ to the monodromy data is canonical nor have we identified the appropriate angle variables in the new coordinates.

\section{Further Discussion}

There is a vast literature on monodromy problems, starting with the investigations of Riemann on analytic functions defined by their branching properties, up to the algebraic geometry studies of recent years (for an introductory survey, see [26]). We have not found modern mathematical work which has concerned itself with the "linearizability" properties of the nonlinear deformation equations which express the monodromy preservation property, excepting, of course, the papers by Satō et al., and the recent thesis of Ueno.

It has been mentioned repeatedly that the work of Satō et al. [7] not only provided the stimulus for the present paper, but also suggests many further problems about singular points and deformation theory. After completing a first draft of this paper, we learned that K. Ueno of the Kyoto University RIMS had carried out investigations [27] which overlap ours to some extent. He derives deformation equations for $n \times n$ systems with singular points of various ranks at $\zeta=0, \infty$, and establishes results of the kind contained in Sect. 3C above. In other respects, his work and ours are complementary. He has not yet considered the inverse problem; on the other hand, he had found a remarkable generalization of our rational-solution example in $3 \mathrm{~F}$. By including apparent singularities in equations such as (3.2) or (4.2), i.e., by including a term

$$
\sum_{1}^{N} \frac{H_{j}}{\zeta-\alpha_{j}}
$$

with certain assumptions about the indicial roots at the $\alpha_{j}$, he can recover the $N$-soliton solutions of $M K d V$, sine-Gordon, etc. (an appropriate limit, in which all 
$\alpha_{j} \rightarrow 0$, should yield the rational solutions). This is the clearest evidence yet that soliton and self-similar solutions will fit into a unified framework.

As we expect that monodromy-preserving deformations will play an increasingly important role in diverse problems of physics and applied mathematics, we now give a brief description of some of the other papers we have found particularly useful. We also outline various reformulations of the inverse problem discussed in earlier sections, in order to emphasize the similarities with well-established and successful approaches to other inverse spectral problems.

The pioneering work on irregular-singular Riemann-Hilbert problems is that of Birkhoff $[16,17]$. In [16], Birkhoff analyzed the behavior of certain canonical equations near an irregular singular point, counted the number of characteristic parameters of the asymptotic solutions and showed it to equal the number of adjustable coefficients of the differential equation, and hence suggested the possibility of solving the inverse problem. In our formulation of the problem, we have been guided more by inverse scattering than by Birkhoff's solution, which we have found to be inaccessible on certain points.

While the structure of solutions near an irregular singular point has been the object of many studies since Birkhoff's influential papers, the problem of finding equations with prescribed Stokes multipliers is encountered only rarely. The extensive work of Sibuya [28] should be mentioned in this connection; its relevance to deformation problems is still unexplored. The work of Sato et al. is obviously relevant to problems of irregular singular monodromy; except for a brief comment in [7, III], however, they have not developed this aspect of their theory. Their work shows that irregular singular points of ordinary differential equations can be transformed to regular singular points of partial differential equations. Indeed, the idea suggested by their approach, to use partial differential equations to find representations of and to investigate solutions of ordinary differential equations with irregular singular points, has not yet been explored and seems to be a fruitful area for study.

The irregular-singular Riemann-Hilbert problem fits quite naturally into the inverse spectral approaches developed for the solution of nonlinear evolution equations. We briefly describe the various connections.

In [29], Zakharov and Shabat solve the inverse scattering problem as follows. Define solutions $\hat{\phi}, \hat{\psi}$ of

$$
\begin{aligned}
& v_{1 x}+i \zeta v_{1}=q v_{2} \\
& v_{2 x}-i \zeta v_{2}=q v_{1}
\end{aligned}
$$

by

$$
\hat{\phi} \sim\left(\begin{array}{l}
1 \\
0
\end{array}\right) e^{-i \zeta x}, x \rightarrow-\infty, \hat{\psi} \sim\left(\begin{array}{l}
0 \\
1
\end{array}\right) e^{i \zeta x}, x \rightarrow+\infty .
$$

Then $\hat{\phi}=a \hat{\bar{\psi}}+b \hat{\psi}$, where $\hat{\bar{\psi}}=\left(\hat{\psi}_{2}^{*}\left(x, \zeta^{*}\right), \hat{\psi}_{1}^{*}\left(x, \zeta^{*}\right)^{T}(q\right.$ is real $)$. Set

$$
\Phi(\zeta, x)=\left\{\begin{array}{l}
a^{-1}(\zeta) \hat{\phi}(x, \zeta) e^{i \zeta x}, \operatorname{Im} \zeta>0 \\
\left(\begin{array}{l}
\hat{\psi}_{2}^{*}\left(x, \zeta^{*}\right) \\
\hat{\psi}_{1}^{*}\left(x, \zeta^{*}\right)
\end{array}\right) e^{i \zeta x}, \operatorname{Im} \zeta<0 .
\end{array}\right.
$$


From the jump of $\Phi$ across the real axis,

$$
\eta(\xi, x) \equiv \Phi(\xi+i 0, x)-\Phi(\xi-i 0, x),
$$

reconstruct $\Phi$ by a Cauchy integral; this leads to a system of singular integral equations for $\hat{\psi}$.

One can reword this approach. For $\operatorname{Im} \zeta>0, \Phi(\zeta, x)$ has an asymptotic expansion $\left(\begin{array}{l}1 \\ 0\end{array}\right)+\sum \Phi_{n} \zeta^{-n}$. The analytic continuation of this expansion to $\operatorname{Im} \zeta<0$ is not the expansion of an analytic continuation of $\Phi$ from $\operatorname{Im} \zeta>0$; rather, it is the expansion of $\Phi$ as defined by (6.2) in $\operatorname{Im} \zeta<0$. The jump $(6.3), \eta(\zeta, x)$, is given by

$$
\frac{b(\zeta)}{a(\zeta)} e^{i \zeta x} \hat{\psi}(\zeta, x)
$$

it is analogous to, and has the form of, a product of a "Stokes multiplier" and a "recessive solution". In contrast to the cases discussed in the present paper, however, $\frac{b}{a}(\zeta)$ has a very general dependence on $\zeta$. The reason is that $\hat{\phi}(\zeta, x)$ and $\hat{\psi}(\zeta, x)$ do not satisfy differential equations in $\zeta$ with polynomial coefficients. When they do [if $q$ evolves in a self-similar manner, or if the $t$ dependence is dropped in (A.4)], the reflection coefficient has the form of the product of $\frac{b}{a}(0)$ and $\exp 8 i \zeta^{3} / 3$. Appropriate scaling of the solutions $\hat{\phi}$ and $\hat{\psi}$ with the factors $\exp \pm 4 i \zeta^{3} / 3$ then shows the jump (6.4) to be the product of a Stokes multiplier and a recessive solution.

Zakharov [30] has recently propounded an extension of inverse-scattering ideas, based on Riemann-Hilbert problems. This generalizes the Zakharov-Shabat method sketched above, in that the eigenfunctions are reconstructed from prescribed jumps across arbitrary closed curves in the $\zeta$-plane. Whereas Zakharov has formulated his new method in extreme generality, the only solutions published so far have a soliton character, in that they correspond to point spectra of certain operators. Our paper, from this perspective, provides the first other type of solution derivable by ideas related to Zakharov's and not accessible to inverse scattering. The curves across which the "jumps" are prescribed are more complicated in our examples than is envisaged in [30].

It is interesting that the irregular monodromy preserving deformations relate as naturally to periodic inverse spectral theory as they do to scattering theory. The approach developed by Krichever ([31], see also Novikov [32]) is particularly relevant. Give

i) a Riemann surface $S$, of genus $g$, with a point called $\infty$,

ii) a nonspecial divisor $P_{1}+\ldots+P_{g}$,

and seek a function $\psi(x, t, y, P)$, meromorphic for $P \in S$, except at $P=\infty$, such that

iii) $\psi \sim \exp (k x+R(k) t+Q(k) y)$ near $k=\infty\left(\frac{1}{k}\right.$ is the local parameter at $\left.\infty\right)$,

iv) $\psi(0,0,0, P)=1$,

v) the poles of $\psi$ are at $P_{1}, \ldots, P_{g}$, independently of $x, t, y$. 
There is a unique such function; it is analogous to the Bloch eigenfunction of a differential operator with periodic coefficients. The coefficients in the asymptotic expansion of $\psi$ at $k=\infty$ are functions of $x, t, y$, from which solutions of a certain Zakharov-Shabat equation

$$
L_{t}-M_{y}=[L, M]
$$

can be constructed.

These requirements determine a holomorphic line bundle over the Riemann surface $S$. The transition functions of this bundle are defined in the finite part of $S$ from functions which locally realize the divisor (ii), and at $\infty$ by the function (iii). The bundle depends on $x, t, y$ because of this construction at $\infty$, and it turns out that the variation with $x, t, y$ is linear in the space of moduli of line bundles, i.e. the Jacobian variety of $R$. This leads to the well-known linearization of isospectral flows on the Jacobian, by means of $\theta$-functions.

The Stokes multiplier problem suggests an analogous construction. In connection with (3.2), for example, we cover the complex plane by six slightly overlapping sectors. To each point, we attack the group $\operatorname{SL}(2, \mathbb{C})$. The transition functions of an SL $(2, \mathbb{C})$ principal bundle are defined by the Stokes matrices (3.12), with some modifications to incorporate a $v \neq 0$ branch point or a $v=n$ pole. This construction is not really useful until the discontinuity of Stokes jumps at $\infty$ can be resolved; only a bundle over a non-contractible surface will carry nontrivial geometric information. Nevertheless, the idea is suggestive and is currently under investigation. We expect that the bundle will vary with the coefficients $x, t, \ldots$ in the exponent $\theta(\zeta)$; this geometric interpretation should make quite clear that in any deformation problem, the coefficients of $\theta(\zeta)$ are to be regarded as independent variables. In particular, our method is applicable to classes of nonlinear, nonautonomous equations in several independent variables (some examples are given in a report to appear in the Proceedings of the 1979 US-USSR Symposium on Solitons held in Kiev).

Acknowledgments. The authors are grateful for support by NSF grants MCS75-07548 A01, MPS7507530, MCS 79-03533, ONR grant N00014-76-C-0867, and US Army Contract DAAG29-78-G-0059.

H. Flaschka thanks Prof. M. Satō, Drs. T. Miwa and M. Jimbo, and Mr. K. Ueno for many stimulating discussions; these were made possible by a US-Japan Cooperative Science Program grant, and by the kind hospitality of the Kyoto University RIMS in January 1978 and April 1979.

\section{Appendix I}

Here we quote results published in reference [33]. It was shown there that the most general equation with $x$-dependent coefficients which can be solved by the inverse scattering transform associated with the $n^{\text {th }}$ order system

$$
\begin{gathered}
\quad V_{x}=\left(\zeta R_{0}+P(x, t)\right) V, \\
R_{0}=\left(\beta_{j} \delta_{i j}\right), \beta_{i} \neq \beta_{j}, P=\left(p_{i j}\right), p_{i i}=0, V \text { an } n \text {-vector, is } \\
G\left(D_{R}, t\right) P_{t}=\Omega\left(D_{R}, t\right)[C, P]+F\left(D_{R}, t\right) x\left[R_{0}, P\right] .
\end{gathered}
$$

In (A.2), $G, \Omega$, and $F$ are entire functions of an integro-differential operator $D_{R}, C$ is a diagonal matrix and the bracket denotes the commutator. The operator $D_{R}$, 
which is only applied to off-diagonal matrices $H_{F}\left(h_{l m}\right), h_{l l}=0$, is defined as follows. First introduce the matrix $\left(H_{F}\right)_{R}=\left(\frac{h_{l m}}{i\left(\beta_{l}-\beta_{m}\right)}\right)$, the solution of $\left[R_{0},\left(H_{F}\right)_{R}\right]=H_{F}$. The diagonal counterpart of $H_{F}$ is defined to be $H_{D}=-\int_{\infty}^{x}\left[H_{F}, P\right]_{D} d y$. Secondly, define the operator $D$ acting on $H_{F}$ to be

$$
\begin{aligned}
D H_{F} & =\frac{\partial}{\partial x} H_{F}+\left[H_{F}, P\right]_{F}+\left[\int_{x}^{\infty}\left[H_{F}, P\right]_{D} d y, P\right] \\
& =\frac{\partial}{\partial x} H+[H, P], H=H_{F}+H_{D} .
\end{aligned}
$$

The subscripts $F$ and $D$ in (A.3) denote the off-diagonal and diagonal parts of the designated matrices, respectively. Finally, $D_{R} H_{F}=D\left(H_{F}\right)_{R}$. We use the lower limit $\infty$ in the definition of $H_{D}$ in a formal manner, to indicate that we simply ignore the constant of integration. The corresponding $t$ and $\zeta$ dependence of $V(x, t, \zeta)$ is given by

$$
G(\zeta, t) V_{t}+F(\zeta, t) V_{\zeta}=\left(x F R_{0}+(Q+S-T)\right) V .
$$

The quantities $Q, S$, and $T$ are defined as follows. Let $\Omega\left(D_{R}, t\right)=\sum_{0} \omega_{m}(t) D_{R}^{m}$, then $Q=\sum \omega_{m} Q^{(m)}$ where $Q^{(m)}=Q_{m}+Q_{m-1} \zeta+\ldots+Q_{1} \zeta^{m-1}+C \zeta^{m}$, and

$$
Q_{S F}=\left(D_{R}^{S-1}[C, P]\right)_{R}, \quad S=1, \ldots, m,
$$

$$
\begin{aligned}
& Q_{S D}=\int_{x}^{\infty}\left[Q_{S F}, P\right]_{D} d y, \\
& Q_{S}=Q_{S F}+Q_{S D} .
\end{aligned}
$$

Define the sequences $\left\{T_{k}\right\},\left\{S_{l}\right\}$ in a similar way;

$$
\begin{aligned}
& T_{k F}=\left(D_{R}^{k-1} P_{t}\right)_{R}, T_{k D}=\int_{x}^{\infty}\left[T_{k F}, P\right]_{D} d y, T_{k}=T_{k F}+T_{k D}, k \geqq 1, \\
& S_{l F}=\left(D_{R}^{l-1} x\left[R_{0}, P\right]\right)_{R}, S_{l D}=\int_{x}^{\infty}\left[S_{l F}, P\right]_{D} d y, S_{l}=S_{l F}+S_{l D}, l \geqq 1 .
\end{aligned}
$$

Then if $G=\sum g_{k}(t) D_{R}^{k}$ and $F=\sum f_{l}(t) D_{R}^{l}$,

$$
\begin{array}{ll}
T=\sum g_{k}(t) T^{(k)}, & T^{(k)}=T_{k}+T_{k-1} \zeta+\ldots+T_{1} \zeta^{k-1}, \\
S=\sum f_{l}(t) S^{(l)}, & S^{(l)}=S_{l}+S_{l-1} \zeta+\ldots+S_{1} \zeta^{l-1} .
\end{array}
$$

Example 1. Let us look at the case where $n=2, G=1, F=f_{1} D_{R}, \Omega=\omega_{3} D_{R}^{3}$, $P=q\left(Y_{2}+Y_{3}\right), \quad R_{0}=C_{0}=-i Y_{1}, \quad Y_{1}=\left(\begin{array}{cc}1 & 0 \\ 0-1\end{array}\right), \quad Y_{2}=\left(\begin{array}{ll}0 & 1 \\ 0 & 0\end{array}\right), \quad Y_{3}=\left(\begin{array}{ll}0 & 0 \\ 1 & 0\end{array}\right)$. Then $T=0 ; S=f_{1} S^{(1)}=f_{1} S_{1}=f_{1} x q\left(Y_{2}+Y_{3}\right) ; Q=\omega_{3} Q^{(3)}=\omega_{3}\left(Q_{3}+Q_{2} \zeta+Q_{1} \zeta^{2}-i Y_{1} \zeta^{3}\right) ;$ $Q_{1}=q\left(Y_{2}+Y_{3}\right), Q_{2}=\frac{i}{2} q_{x}\left(Y_{2}-Y_{3}\right)-\frac{i}{2} q^{2} Y_{1}, Q_{3}=\frac{-1}{4}\left(q_{x x}-2 q^{3}\right)\left(Y_{2}+Y_{3}\right)$. Then, 
the Eq. (A.2) is

$$
q_{t}=\frac{-\omega_{3}}{4}\left(q_{x x}-2 q^{3}\right)_{x}+f_{1}(x q)_{x} .
$$

Note that the Painleve equation can be found by either taking $f_{1}=0$ and looking for the solution $q(x, t)=\frac{1}{(3 t)^{1 / 3}} \hat{q}\left(\frac{x}{(3 t)^{1 / 3}}\right)$ or by simply setting $f_{1}=1$ and ignoring the $t$-dependence altogether. In fact, all the self-similar solutions of evolution equations with $F=0$ can be obtained by taking appropriate functions $F$ and ignoring the $t$-dependence. Moreover, there are many equations which are solvable by the method introduced in this paper and which are not the result of a self-similar transformation on a solvable evolution equation. Ignoring the $t$ dependence, the $\zeta$ and $x$ equations for $V$ are precisely (3.2) and (3.3) respectively. Note that the term $Q_{3}+S_{1}$ vanishes by virtue of (3.1).

Example 2. We present an equation solvable by our method which is not obtained as a self-similar limit of an evolution equation. Let $R_{0}=C=-i Y_{1}, P=q Y_{2}+r Y_{3}$, $F=\frac{1}{4}, \Omega=D_{R}^{2}, r=-q^{*}$; we obtain $q_{x x}+2 q^{2} q^{*}-x q=0$. When our method is applied to this equation, the details are similar to those introduced in Sect. 3.

Example 3. We show that (4.1) is the integrability condition for (4.1), (4.2). Take $n=2, \Omega=1, G=0, F=-D_{R}^{2}, R=C=-i Y_{1}, P=q\left(Y_{2}+Y_{3}\right)$ and $q=-\frac{u_{x}}{2}$. Then Eq. (A.2) reads

$$
\left(x u_{x}\right)_{x}=-4 \sinh u .
$$

We also find that $Q=-i Y_{1}, S=-S_{2}-\zeta S_{1}=\frac{x u_{x}}{2}\left(Y_{2}+Y_{3}\right)-i \sinh u\left(Y_{2}-Y_{3}\right)$

$+i(1-\cosh u) Y_{1}$. Therefore, (A.1) is

$$
\begin{aligned}
& v_{1 x}=-i \zeta v_{1}+q v_{2} \\
& v_{2 x}=q v_{1}+i \zeta v_{1}
\end{aligned}
$$

and (A.4) is

$$
\begin{aligned}
& v_{1 \zeta}=\left(-i x+\frac{i}{\zeta^{2}} \cosh u\right) v_{1}+\left(\frac{i}{\zeta^{2}} \sinh u-\frac{1}{2 \zeta} x u_{x}\right) v_{2} \\
& v_{2 \zeta}=\left(-\frac{i}{\zeta^{2}} \sinh u-\frac{1}{2 \zeta} x u_{x}\right) v_{1}+\left(i x-\frac{i}{\zeta^{2}} \cosh u\right) v_{2} .
\end{aligned}
$$

\section{Appendix II: The Painlevé II Family}

It is well known [23] that the MKdV equation is only one of an infinite family of equations, all of which are solvable by the scattering problem (2.12). The equations of this family can be derived from Hamiltonians [24],

$$
q_{t}=\frac{\partial}{\partial x} \frac{\delta H_{2 j+1}}{\delta q} .
$$


It is easily shown that a self-similar solution of the form

$$
t^{-\frac{1}{2 j+1}} f\left(x t^{-\frac{1}{2 j+1}}\right)
$$

exists. The resulting ordinary differential equation for $f$ is again the condition that a deformation of a system like (3.2) be monodromy preserving; this time, the asymptotic expansion of $\Psi$ will, of course, involve $e^{ \pm \theta}$ with $\theta=i\left(\omega_{2 j+1} \frac{\zeta^{2 j+1}}{2 j+1}+\zeta x\right)$. The general autonomous equation of the MKdV family,

$$
q_{t}=\frac{\partial}{\partial x} \frac{\delta H}{\delta q}
$$

where

$$
H=\sum_{0}^{N} c_{j} H_{2 j+1}
$$

with constant $c_{j}$, does not have self-similar solutions, since the $H_{2 j+1}$ scale differently. If the $c_{j}$ are time-dependent, however,

$$
c_{j}=\stackrel{\circ}{c}_{j} t^{\frac{2 j+1}{2 N+1}}
$$

then a self-similar solution exists, and is associated with the monodromy for a system $\Psi_{\zeta}=Q \Psi$ which gives rise to expansions involving $e^{ \pm \theta}$,

$$
\theta=i\left(\sum_{0}^{N} \omega_{j} \frac{\zeta^{2 j+1}}{2 j+1}+\zeta x\right) .
$$

This is the Painlevé II family of nonlinear ordinary differential equations. It is apparent that all these equations can be reduced to a system of linear singular integral equations according to the pattern described earlier. Although the solution method parallels that of Sect. 3, the details are too cumbersome to reproduce here. It is possible, however, to give compact formulae for the rational solutions of all these equations (Stokes multipliers zero, and an $n$-th order pole at $\zeta=0)$. This was done in Sect. $3 \mathrm{~F}$.

We note that the equations of the Painleve II family, which we designate as (PII, $n$ ) can be generated very quickly by the formalism described in Appendix I. We take $R_{0}=C=-i Y_{1}, P=q\left(Y_{2}+Y_{3}\right)$. Then the first few equations are:

$$
(\mathrm{PII}, 0): \omega_{1} D_{R}[C, P]+D_{R} x\left[R_{0}, P\right]=0 ;
$$

this integrates to

$$
\left(\omega_{1}+x\right) q=v
$$

and

$$
\begin{aligned}
& \theta(\zeta)=i \omega_{1}+i \zeta x . \\
& (\mathrm{PII}, 1): \omega_{3} D_{R}^{3}[C, P]+\omega_{1} D_{R}[C, P]+D_{R} x\left[R_{0}, P\right]=0,
\end{aligned}
$$

which integrates to

$$
-\frac{\omega_{3}}{4}\left(q_{x x}-2 q^{3}\right)+\left(\omega_{1}+x\right) q=v,
$$


with

$$
\begin{aligned}
& \theta(\zeta)=i \omega_{3} \frac{\zeta^{3}}{3}+i \omega_{1} \zeta+i \zeta x \\
& (\mathrm{PII}, 2): \omega_{5} D_{R}^{5}[C, P]+\omega_{3} D_{R}^{3}[C, P]+\omega_{1} D_{R}[C, P]+D_{R} x\left[R_{0}, P\right]=0,
\end{aligned}
$$

which integrates to

$$
\begin{aligned}
& \frac{-5 \omega_{5}}{16}\left[\left(q_{x x}-2 q^{3}\right)_{x x}-2 q\left(2 q q_{x x}-q_{x}^{2}-3 q^{4}\right)\right] \\
& -\frac{\omega_{3}}{4}\left(q_{x x}-2 q^{3}\right)+\left(\omega_{1}+x\right) q=v
\end{aligned}
$$

with

$$
\theta(\zeta)=\frac{i \omega_{5} \zeta^{5}}{5}+i \omega_{3} \frac{\zeta^{3}}{3}+i \omega_{1} \zeta+i \zeta x
$$

The general equation

$$
\text { (PII, } n): \sum_{r=0}^{N} \omega_{2 r+1} D_{R}^{2 r+1}[C, P]+D_{R} x\left[R_{0}, P\right]=0
$$

has $\theta(\zeta)$ given by (A.12).

\section{Appendix III}

Here we give the explicit connection between the formulae of Satō et al. and Eqs. (4.2), (4.3). In [7, III] it is shown that the basis $\mathbf{W}$, to which we have already referred in the introduction, satisfies a holonomic system of differential equations. Applying a formal Laplace transform (transform variable $u$ ), Satō et al. obtain a system of ordinary differential equations in $u$,

$$
\left(u \frac{d}{d u}+m A u-G^{-1} m \bar{A} G u^{-1}+F\right) \hat{w}(u)=0 .
$$

The dependence of $\hat{w}$ on the parameters $\left(a_{j}, \bar{a}_{j}\right), j=1, \ldots, k$, at which points the solutions of the underlying Dirac equation have multivalued behavior, is provided by an auxiliary set of equations. We now write down this system when $n=2$ and $a_{1}-a_{2}=\frac{t e^{i \theta}}{2 m}$. Then using $G, \bar{G}, F$ as given in $[7$, III $]$ and taking $l_{1}=l_{2}=l=0, \frac{f_{+}}{\varepsilon \kappa^{-1}}$ $=\frac{f_{-}}{\bar{\varepsilon} \kappa}=\frac{f}{2}=\frac{t \psi_{t}}{2}$, we find

$$
\begin{aligned}
& \hat{w}_{u}=\left(-\frac{t}{4}\left(\begin{array}{rr}
1 & 0 \\
0 & -1
\end{array}\right)+\frac{t}{4 u^{2}}\left(\begin{array}{cc}
C & \varepsilon \kappa^{-1} S \\
-\bar{\varepsilon} \kappa S & -C
\end{array}\right)-\frac{1}{u}\left(\begin{array}{cc}
0 & f_{+} \\
f_{-} & 0
\end{array}\right)\right) \hat{w}, \\
& \hat{w}_{t}=\left(-\frac{u}{4}\left(\begin{array}{rr}
1 & 0 \\
0 & -1
\end{array}\right)-\frac{1}{4 u}\left(\begin{array}{cc}
C & \varepsilon \kappa^{-1} S \\
-\bar{\varepsilon} \kappa S & -C
\end{array}\right)-\frac{1}{t}\left(\begin{array}{cc}
0 & f_{+} \\
f_{-} & 0
\end{array}\right)\right) \hat{w},
\end{aligned}
$$


where $C=\cosh 2 \psi, S=\sinh 2 \psi$. By scaling the components $\hat{w}_{1}$ and $\hat{w}_{2}$ by $\sqrt{\bar{\varepsilon} \kappa}$ and $\sqrt{\varepsilon \kappa^{-1}}$, respectively, and using $\varepsilon \bar{\varepsilon}=1$, we can eliminate the factors $\varepsilon \kappa^{-1}$ and $\bar{\varepsilon} \kappa$. Then, introducing the transformation $x=-\frac{1}{16} t^{2}, u=\frac{-i}{4} \zeta t$, in which case $f=t \psi_{t}=x v_{x}($ where $v=2 \psi)$, we find

$$
\begin{aligned}
& \hat{w}_{x}=\left(-i \zeta\left(\begin{array}{rr}
1 & 0 \\
0 & -1
\end{array}\right)+\left(\begin{array}{ll}
0 & q \\
q & 0
\end{array}\right)\right) \hat{w}, \\
& \hat{w}_{\zeta}=\left(-i x\left(\begin{array}{rr}
1 & 0 \\
0 & -1
\end{array}\right)-\frac{x v_{x}}{2 \zeta}\left(\begin{array}{ll}
0 & 1 \\
1 & 0
\end{array}\right)+\frac{i}{\zeta^{2}}\left(\begin{array}{rr}
\cosh v & \sinh v \\
-\sinh v & -\cosh v
\end{array}\right)\right) \hat{w}
\end{aligned}
$$

with $q=\frac{-v_{x}}{2}$. These are our Eqs. (A.6), (A.7), and (4.2) and (4.3).

\section{Appendix IV. The Inversion Equations for PII When $v \neq n$}

Following the ideas outlined in $3 \mathrm{E}$, we can find the equations analogous to (3.33), (3.35) when $v \neq n$. For $\zeta \in S_{1}$, they are:

$$
\begin{aligned}
& \psi^{(2)}(\zeta) e^{-\theta(\zeta)}=\left(\begin{array}{l}
0 \\
1
\end{array}\right)-\frac{1}{2 \pi i} \int_{C} \frac{\psi^{(2)} e^{-\theta}}{\xi-\zeta} d \xi+\frac{b}{2 \pi i} \int_{C_{53}} \frac{\psi^{(1)} e^{-\theta}}{\xi-\zeta} d \xi+\frac{c}{2 \pi i} \int_{C_{57}} \frac{\psi^{(1)} e^{-\theta}}{\xi-\zeta} d \xi \\
&+\frac{a b}{2 \pi i} \int_{C_{53}} \frac{\psi^{(2)} e^{-\theta}}{\xi-\zeta} d \xi+\frac{s}{2 \pi i} \int_{C_{57}} \frac{\psi_{2}^{(1)} e^{-\theta}}{\xi-\zeta} d \xi, \\
& \psi^{(1)}(\zeta) e^{\theta(\zeta)=}=\left(\begin{array}{l}
1 \\
0
\end{array}\right)-\frac{1}{2 \pi i} \int_{C} \frac{\psi^{(1)} e^{\theta}}{\xi-\zeta} d \xi+\frac{a}{2 \pi i} \int_{C_{42}} \frac{\psi^{(2)} e^{\theta}}{\xi-\zeta} d \xi+\frac{b+s}{2 \pi i} \int_{C_{46}} \frac{\psi^{(2)} e^{\theta}}{\xi-\zeta} d \xi \\
&-\frac{b c}{2 \pi i} \int_{C_{46}} \frac{\psi^{(1)} e^{\theta}}{\xi-\zeta} d \xi+\frac{s}{2 \pi i} \int_{C_{67}} \frac{\psi_{3}^{(2)} e^{\theta}}{\xi-\zeta} d \xi .
\end{aligned}
$$

In (A.20) and (A.21), $C$ is a contour originating at $\xi=\infty$, travelling on top of the branch cut along the positive real $\xi$ axis, circling the origin and returning to $\xi=\infty \exp 2 \pi i$ along the lower edge of the branch cut. The parameters $s=2 i \sin v \pi$ and $\psi_{2}^{(1)}, \psi_{3}^{(2)}$ are defined in terms of $\psi^{(1)}, \psi^{(2)}$ by $(3.12)$. Note that the terms involving the contour $C$ simply become $-\operatorname{Res}_{\xi=0} \frac{\psi^{(j)} e^{ \pm \theta}}{\xi-\zeta}, j=1,2$, when $v=n$ as in this case $\psi^{(j)}$ is single valued.

\section{References}

1. Ince, E.L.: Ordinary differential equations. New York: Dover Publications 1947

2. Ablowitz, M.J., Segur, H.: Phys. Rev. Lett. 38, 1103-1106 (1977)

3. Airault, H.: Rational solutions of Painlevé equations (to appear)

4. Wu, T.T., McCoy, B.M., Tracy, C.A., Barouch, E.: Phys. Rev. B 13, 316-371 (1976)

5. Barouch, E., McCoy, B.M., Wu, T.T.: Phys. Rev. Lett. 31, 1409-1411 (1973)

6. McCoy, B.M., Tracy, C.A., Wu, T.T.: J. Math. Phys. 18, 1058-1092 (1977) 
7. Satō, M., Miwa, T., Jimbo, M.: A series of papers entitled Holonomic Quantum Fields: I. Publ. RIMS, Kyoto Univ. 14, 223-267 (1977); II. Publ. RIMS, Kyoto Univ. 15, 201-278 (1979); III. Publ. RIMS Kyoto Univ. 15, 577-629 (1979). IV., V. RIMS Preprints 263 (1978), and 267 (1978). The paper we refer to most often is III. See also a series of short notes: Studies on holonomic quantum fields. I-XV

8. Gardner, C.S., Greene, J.M., Kruskal, M.D., Miura, R.M. : Commun. Pure Appl. Math. 27, 97-133 (1976)

9. Fuchs, R.: Math. Ann. 63, 301-321 (1906)

10. Ablowitz, M.J., Segur, H.: Stud. Appl. Math. 57, 13-44 (1977)

11. Hastings, S.P., McLeod, J.B.: Univ. of Wisconsin, MRC Report No. 1861 (1978)

12. Ablowitz, M.J., Ramani, A., Segur, H.: (i) Lett. Nuovo Cimento 23, 333 (1978). (ii) Two preprints: A connection between nonlinear evolution equations and ordinary differential equations of $P$-type, I, II

13. Tracy, C.A.: Proc. NATO Advanced Study Institute on: Nonlinear equations in physics and mathematics, 1978, (ed. A. Barut). Dordrecht, Holland: Reidel 1978

14. Schlesinger, L.: J. Reine Angewandte Math. 141, 96-145 (1912)

15. Garnier, R.: Ann. Ec. Norm. Sup. 29, 1-126 (1912)

16. Birkhoff, G.D.: Trans. AMS 10, 436-470 (1909)

17. Birkhoff, G.D.: Proc. Am. Acad. Arts Sci. 49, 521-568 (1913)

18. Garnier, R.: Rend. Circ. Mat. Palermo, 43, 155-191 (1919)

19. Davis, H.T.: Introduction to nonlinear differential and integral equations. New York: Dover Publications 1962

20. Choodnovsky, D.V., Choodnovsky, G.V.: Completely integrable class of mechanical systems connected with Korteweg-deVries and multicomponent Schrodinger equations. I. Preprint, École Polytechnique, 1978

21. Moser, J., Trubowitz, E.: (to appear)

22. Olver, F.W.J.: Asymptotics and special functions. New York: Academic Press 1974

23. Ablowitz, M.J., Kaup, D.J., Newell, A.C., Segur, H.: Stud. Appl. Math. 53, 249-315 (1974)

24. Flaschka, H., Newell, A.C.: Springer Lecture Notes in Physics 38, 355-440 (1975)

25. Airault, H., McKean, Jr., H.P., Moser, J.: Comm. Pure Appl. Math. 30, 95-148 (1977)

26. Brieskorn. E.: Jber. Dt. Math.-Verein. 78, 93-112 (1976)

27. Ueno, K.: Kyoto, RIMS master's thesis, Dec. 1978. RIMS Preprints 301, 302 (1979)

28. Sibuya, Y.: (i) Proc. Int. Conf. Diff. Eq. pp. 709-738. (ed. H. A. Antosiewicz). New York: Academic Press 1975; (ii) Bull. AMS 83, 1075-1077 (1977)

29. Zakharov, V.E., Shabat, A.B.: Sov. Phys. JETP 34, 62-69 (1972)

30. Zakharov, V.E.: Paper at I. G. Petrovskii Memorial Converence, Moscow State Univ., Jan. 1976 (this paper has been referred to in many subsequent publications, but has apparently never been published)

31. Krichever, I.M.: Funkts. Anal. Prilozen 11, 15-31 (1977)

32. Novikov, S.P.: Rocky Mt. J. Math. 8, 83-94 (1978)

33. Newell, A.C.: Proc. Roy. Soc. London A 365, 283-311 (1979)

Communicated by A. Jaffe

Received July 29, 1979; in revised form March 19, 1980 